\title{
On the Number of Zeros of Linear Combinations of Independent Characteristic Polynomials of Random Unitary Matrices
}

\author{
Yacine Barhoumi-Andréani ${ }^{1}$, Christopher Hughes ${ }^{2}$, \\ Joseph Najnudel ${ }^{3}$, and Ashkan Nikeghbali ${ }^{4}$ \\ ${ }^{1}$ Department of Statistics, University of Warwick, Coventry CV4 7AL, UK, \\ ${ }^{2}$ Department of Mathematics, University of York, York YO10 5DD, UK, \\ ${ }^{3}$ Institut de Mathématiques de Toulouse, Université Paul Sabatier, 118 \\ route de Narbonne, F-31062 Toulouse Cedex 9, France, and ${ }^{4}$ Institut für \\ Mathematik, Universität Zürich, Winterthurerstrasse 190, 8057 Zürich, \\ Switzerland
}

Correspondence to be sent to: christopher.hughes@york.ac.uk

We show that for any linear combination of characteristic polynomials of independent random unitary matrices with the same determinant, the expected proportion of zeros lying on the unit circle tends to 1 as the dimension of the matrices tends to infinity. This result is the random matrix analog of an earlier result by Bombieri and Hejhal on the distribution of zeros of linear combinations of $L$-functions, and thus is consistent with the conjectured links between the value distribution of the characteristic polynomial of random unitary matrices and the value distribution of $L$-functions on the critical line.

\section{Introduction}

Over the past two decades, there have been many new results at the interface of random matrix theory and analytic number theory that can be considered as evidence for the zeros of the Riemann zeta function being statistically distributed as eigenvalues of large 
random matrices (matrices from the Gaussian Unitary Ensemble, or Haar-distributed unitary matrices). The interested reader can refer to [14, 20, 21] for a detailed account with many references, and to [13] for the function field framework. Since the seminal papers by Keating and Snaith [15, 16], it is believed that the characteristic polynomial of a random unitary matrix on the unit circle provides a very accurate model for the value distribution of the Riemann zeta function (or, more generally, $L$-functions) on the critical line. This analogy was used by Keating and Snaith to produce the moments conjecture and since then the characteristic polynomial has been the topic of many research papers, and the moments of the characteristic polynomial have now been derived with many different methods: representation theoretic approach (see [6, 7]); super-symmetry (see [20]); analytic methods (such as Toeplitz determinants [20] and orthogonal polynomials on the unit circle [17]); and probabilistic techniques [4]. Each method brings a new insight to the problem. Many more fine-grained properties of the characteristic polynomial have been established (e.g., the large deviations principle [10], local limit theorems [18], the analog of the moments conjecture for finite field zeta functions [1, 12], etc.). Moreover, thanks to this analogy, one has been able to perform calculations in the random matrix world (whose analog in the number theory world appears to be currently out of reach) to produce conjectures for the analogous arithmetic objects (see [23] for a recent account).

There are nonetheless certain results that can be proved in both worlds, such as Selberg's central limit theorem for the Riemann zeta function and the Keating-Snaith central limit theorem for the characteristic polynomial of random unitary matrices (see [15]). In fact, Selberg's central limit theorem can be proved more generally for a wide class of $L$-functions (see [3, 22]).

Roughly speaking, an $L$-function must be defined by a Dirichlet series for $\operatorname{Re}(s)>1$, have an Euler product (with some growth condition on the coefficients of this product), an analytic continuation (except for finitely many poles all located on the line $\operatorname{Re}(s)=1$ ), and must satisfy a functional equation. Such $L$-functions are expected to satisfy the general Riemann hypothesis (GRH), which says that all the nontrivial zeros are located on the critical line, the line $\operatorname{Re}(s)=\frac{1}{2}$.

Now if one considers a finite number of such $L$-functions, all satisfying the same functional equation, then one can wonder if the zeros of a linear combination of these $L$-functions are still on the critical line. In general, the answer is that GRH does not hold anymore for such a linear combination even though it still has a functional equation (this can be thought of coming from the fact that such a linear combination does not have an Euler product anymore). But Bombieri and Hejhal proved in [3] that nonetheless $100 \%$ of the zeros of such linear combinations are still on the critical line (under 
an extra assumption of "near orthogonality" which ensures that the logarithm of the $L$-functions are statistically asymptotically independent and that $100 \%$ of the zeros of each $L$-function do not occur in clumps). In this paper, we will show that a similar result holds for linear combinations of independent characteristic polynomials of random unitary matrices. The result on the random matrix side is technical and difficult. In addition to being an extra piece of evidence that the characteristic polynomial is a good model for the value distribution of $L$-functions, the result is also remarkable when viewed in the general setting of random polynomials. The main goal of this article is to show that on average, any linear combination of characteristic polynomials of independent random unitary matrices having the same determinant has a proportion of zeros on the unit circle which tends to 1 when the dimension goes to infinity.

More precisely, if $U$ is a unitary matrix of order $N \geqslant 1$, for $z \in \mathbb{C}$ let

$$
\Phi_{U}(z)=\operatorname{det}\left(I_{N}-z U\right)
$$

be the characteristic polynomial of $U$. From the fact that $U$ is unitary, we get the functional equation

$$
\Phi_{U}(z)=(-z)^{N} \operatorname{det}(U) \overline{\Phi_{U}}(1 / z) .
$$

For $z$ on the unit circle, this equation implies that

$$
\Phi_{U}(z)=R(z) \sqrt{(-z)^{N} \operatorname{det}(U)},
$$

where $R(z)$ is real-valued (with any convention taken for the square root). Now, let $\left(U_{j}\right)_{1 \leqslant j \leqslant n}$ be a collection of $n$ unitary matrices of order $N$, and let $\left(b_{j}\right)_{1 \leqslant j \leqslant n}$ be real numbers. We wish to study the number of zeros on the unit circle of the linear combination

$$
F_{N}(z)=\sum_{j=1}^{n} b_{j} \Phi_{U_{j}}(z)
$$

If we want $F$ to have most of its zeros on the unit circle, it is reasonable to expect that we need a "unidimensional condition" for the equation $F(z)=0$ and $|z|=1$, that is, a functional equation similar to the equation satisfied by $U$. This equation obviously exists if all the characteristic polynomials $\Phi_{U_{j}}$ satisfy the same functional equation, which happens when the matrices $U_{j}$ have the same determinant. By symmetry of the unitary group, it is natural to assume that the unitary matrices have determinant 1 . More precisely, the main result of the article is the following theorem. 
Theorem 1.1. Let $\left(b_{j}\right)_{1 \leqslant j \leqslant n}$ be nonzero real numbers. For $N \geqslant 1$, let

$$
F_{N}(z):=\sum_{j=1}^{n} b_{j} \Phi_{U_{j}}(z),
$$

where $\left(U_{j}\right)_{1 \leqslant j \leqslant n}$ is a family of independent matrices whose distribution is the Haar measure on the special unitary group $\mathrm{SU}(N)$. The expected proportion of zeros of $F_{N}$ on the unit circle tends to 1 as $N$ goes to infinity, that is,

$$
\mathbb{E}\left(\left|\left\{z \in \mathbb{U}: F_{N}(z)=0\right\}\right|\right)=N-o(N),
$$

where $\left|\left\{z \in \mathbb{U}: F_{N}(z)=0\right\}\right|$ is the number of $z$ on the unit circle which satisfy $F_{N}(z)=0$

The whole paper is devoted to the proof of this result. Before explaining the strategy of the proof, we make a few remarks.

Remark 1.2. Theorem 1.1 can be stated as

$$
\lim _{N \rightarrow \infty} \mathbb{E}\left(\frac{1}{N}\left|\left\{z \in \mathbb{U}: F_{N}(z)=0\right\}\right|\right)=1 .
$$

Since the random variable $\frac{1}{N}\left|\left\{z \in \mathbb{U}: F_{N}(z)=0\right\}\right|$ is bounded by 1 , the convergence holds in all $L^{p}$ spaces for $p \geqslant 1$. It therefore also holds in probability since convergence in $L^{1}$ implies convergence in probability.

Remark 1.3. The fact that we impose the condition that the matrices have the same determinant is similar to the condition in [3] that the $L$-functions have the same functional equation. Moreover, in our framework, the analog of the Riemann hypothesis is automatically satisfied since all the zeros of each characteristic polynomial are on the unit circle.

Remark 1.4. The fact that the proportion of zeros on the unit circle tends to 1 is a remarkable fact when considered as a result about random polynomials. It is well known that the characteristic polynomial of a unitary matrix is self-inversive. (A self-inversive polynomial is one where $a_{N-k}=\exp (\mathrm{i} \theta) \bar{a}_{k}$ for some $\theta \in \mathbb{R}$, where $\left(a_{k}\right)_{0 \leqslant k \leqslant N}$ are the coefficients of the polynomial.) Self-inversive random polynomials are of interest in the context of semiclassical approximations in quantum mechanics, and determining the proportion of zeros on the unit circle is an important problem in that context. Bogomolny et al. [2] showed that if the first half of the coefficients are chosen as independent complex Gaussian random variables (the second half being fixed by the self-inverse 
symmetry), then asymptotically $\frac{1}{\sqrt{3}}$ of all the zeros are exactly on the unit circle. Hence, we can say that our result is not typical of what is expected for classical random polynomials built from independent Gaussian random variables. In our framework, we do not know the distribution of the coefficients, but we do know that they are not independent. Consequently, the classical methods which use the independence of the coefficients (or use the fact that they are Gaussian if one wishes to add some dependence) do not work here. Using general results on random polynomials whose coefficients are not necessarily independent or equidistributed [11], one can deduce that the zeros cluster uniformly around the unit circle. But showing that they are almost all precisely on the unit circle is a much more refined statement.

We now say a few words about our strategy of proof of Theorem 1.1. In fact, we use the same general method as in [3], called the "carrier waves" method, but the ingredients of our proof are different, in the sense that they are probabilistic. For instance, we use the coupling method, concentration inequalities and the recent probabilistic representation of the characteristic polynomial obtained in [4]. More precisely, for $U \in U(N)$ and $\theta \in \mathbb{R}$, we denote by $Z_{U}(\theta)$ the characteristic polynomial of $U$ evaluated at $\mathrm{e}^{-\mathrm{i} \theta}$, that is, $Z_{U}(\theta)=\Phi_{U}\left(\mathrm{e}^{-\mathrm{i} \theta}\right)$. Then we make a simple transformation of the linear combination $F_{N}$ in order that it is real-valued when restricted as a function on the unit circle:

$$
\mathrm{i}^{N} \mathrm{e}^{\mathrm{i} N \theta / 2} F_{N}\left(\mathrm{e}^{-\mathrm{i} \theta}\right)=\mathrm{i}^{N} \mathrm{e}^{\mathrm{i} N \theta / 2} \sum_{j=1}^{n} b_{j} \Phi_{U_{j}}\left(\mathrm{e}^{-\mathrm{i} \theta}\right)=\sum_{j=1}^{n} b_{j} \mathrm{i}^{N} \mathrm{e}^{\mathrm{i} N \theta / 2} Z_{U_{j}}(\theta) .
$$

Using the fact that $U_{j} \in \mathrm{SU}(N)$, one checks that $\mathrm{i}^{N} \mathrm{e}^{\mathrm{i} N \theta / 2} Z_{U_{j}}(\theta)$ is real, and thus the number of zeros of $F_{N}$ on the unit circle is bounded from below by the number of sign changes, when $\theta$ increases from $\theta_{0}$ to $\theta_{0}+2 \pi$ (with $\theta_{0}$ to be chosen carefully), of the real quantity given by the right-hand side of the equation above. The notion of carrier waves is explained in detail in [3, pp. 824-827] and we do not explain it again but we would rather give a general outline. The main idea is that informally, with "high" probability and for "most" of the values of $\theta$, one of the characteristic polynomials $Z_{U_{j}}$ dominates all the others (it is the "carrier wave"). More precisely, Lemma 3.8 implies the following: If $\delta$ depends only on $N$ and tends to zero when $N$ goes to infinity, then there exists, with probability $1-o(1)$, a subset of $\left[\theta_{0}, \theta_{0}+2 \pi\right)$ with Lebesgue measure $o$ (1) such that for any $\theta$ outside this set, one can find $j_{0}$ between 1 and $n$ such that $|\log | Z_{U_{j_{0}}}(\theta)|-\log | Z_{U_{j}}(\theta)||>\delta \sqrt{\log N}$ for all $j \neq j_{0}$. In other words, one of the terms in the sum of the right-hand side of (1) should dominate all the others. Moreover, Lemma 3.13 informally gives the following: With high probability, the order of 
magnitude of each of the characteristic polynomials does not change too quickly, so if the interval $\left[\theta_{0}, \theta_{0}+2 \pi\right)$ is divided into sufficiently many equal subintervals, the index of the carrier wave remains the same in a "large" part of each subinterval. The zeros of $Z_{U_{j_{0}}}$ correspond to sign changes of $\mathrm{i}^{N} \mathrm{e}^{\mathrm{i} N \theta / 2} Z_{U_{j_{0}}}(\theta)$, and since this is the dominant term of (1), one gets sign changes of $\mathrm{i}^{N} \mathrm{e}^{\mathrm{i} N \theta / 2} F_{N}\left(\mathrm{e}^{-\mathrm{i} \theta}\right)$. By counting all these sign changes, one deduces a lower bound for the number of zeros of $F_{N}$ on the unit circle.

The main issue of the present paper is to make rigorous this informal construction, in such a way that one gets a lower bound $N-O(N)$. One of the reasons why the proof becomes technical and involved is that we have to take into account two different kinds of sets, and show that they have almost "full measure": subsets of the interval $\left[\theta_{0}, \theta_{0}+2 \pi\right)$ and subsets of $\mathrm{SU}(N)$.

Our proof is structured as follows: We first give two standard results (Propositions 2.1 and 2.5), one on the disintegration of the Haar measure on $U(N)$ and the other one which establishes a relationship between the number of eigenvalues in a given fixed arc to the variation of the imaginary part of the logarithm of the characteristic polynomial. (Indeed, most of our results on random matrices are established for $U(N)$ and we show how to go from the results for $U(N)$ to those for $\mathrm{SU}(N)$.) Then we provide some estimates on the real and imaginary parts of the logarithm of the characteristic polynomial (Lemmas 3.1 and 3.2) as well as a bound on the concentration of the law of the logarithm of the characteristic polynomial (Lemma 3.3). Then we provide bounds on the oscillations of the real and imaginary parts of the logarithm of the characteristic polynomial (Lemma 3.7). These estimates, and some further intermediary ones which we establish, are also useful in their own right and complement the existing results in the literature on characteristic polynomials. We then introduce our subdivisions of the interval $\left[\theta_{0}, \theta_{0}+2 \pi\right)$ and the corresponding relevant random sets to implement the carrier waves technique. Finally, we combine all these estimates together to show that the average number of sign changes of (1) is at least $N\left(1-O\left((\log N)^{-1 / 22}\right)\right)$. The exponent $-1 / 22$ does not play any major role in our analysis.

\section{Notation}

We gather here some notation used throughout the paper.

We write $U(N)$ for the unitary group of order $N$, while $\mathrm{SU}(N)$ stands for the subgroup of elements $U(N)$ whose determinant is equal to 1 . We let $\mathbb{P}_{U(N)}$ and $\mathbb{P}_{\mathrm{SU}(N)}$ denote the probability Haar measure on $U(N)$ and $\mathrm{SU}(N)$, respectively. Similarly, we denote by $\mathbb{E}_{U(N)}$ and $\mathbb{E}_{\mathrm{SU}(N)}$ the corresponding expectations. For $n$ a positive integer, we let 
$\mathbb{P}_{\mathrm{SU}(N)}^{(n)}$ be the $n$-fold product of the Haar measure on $\mathrm{SU}(N)$, and $\mathbb{E}_{\mathrm{SU}(N)}^{(n)}$ the corresponding expectation.

If $U$ is a unitary matrix, we write its characteristic polynomial as $\Phi_{U}(z)=$ $\operatorname{det}\left(I_{N}-z U\right)$, with $z \in \mathbb{C}$. For $\theta \in \mathbb{R}$, we denote by $Z_{U}(\theta)$ the characteristic polynomial of $U$ evaluated at $\mathrm{e}^{-\mathrm{i} \theta}$, that is, $Z_{U}(\theta)=\Phi_{U}\left(\mathrm{e}^{-\mathrm{i} \theta}\right)$.

We shall denote the Lebesgue measure on $\mathbb{R}$ by $\lambda$. If $\alpha>0$ is a constant and if $I$ is an interval of length $\alpha$, then $\lambda_{\alpha}$ will denote the normalized measure $\frac{1}{\alpha} \lambda$ on the interval $I$.

If $n$ is an integer, we denote $\llbracket 1, n \rrbracket$ the set of integers $\{1, \ldots, n\}$, and if $\mathcal{E}$ is a finite set, we write $|\mathcal{E}|$ for the number of its elements.

We shall introduce several positive quantities during the proof: $K>0, M>0$, and $\delta>0$. The reader should have in mind that these quantities will eventually depend on $N$. Unless stated otherwise, $N \geqslant 4$ and $K$ is an integer such that $2 \leqslant K \leqslant N / 2$, and $M=N / K$. In the end, we will use $K \sim N /(\log N)^{3 / 64}$ and $\delta \sim(\log N)^{-3 / 32}$.

\section{Some General Facts}

In this section, we state some general facts in random matrix theory, which will be used later on.

\subsection{Disintegration of the Haar measure on unitary matrices}

Proposition 2.1. Let $\mathbb{P}_{U(N)}$ be the Haar measure on $U(N), \mathbb{P}_{S U(N)}$ be the Haar measure on $\mathrm{SU}(N)$, and for $\theta \in \mathbb{R}$, let $\mathbb{P}_{\mathrm{SU}(N), \theta}$ be the image of $\mathbb{P}_{\mathrm{SU}(N)}$ by the application $U \mapsto \mathrm{e}^{\mathrm{i} \theta} U$ from $U(N)$ to $U(N)$. Then, we have the following equality:

$$
\int_{0}^{2 \pi} \mathbb{P}_{\mathrm{SU}(N), \theta} \frac{\mathrm{d} \theta}{2 \pi}=\mathbb{P}_{U(N)},
$$

that is, for any continuous function $F$ from $U(N)$ to $\mathbb{R}_{+}$, the expectation $\mathbb{E}_{\mathrm{SU}(N), \theta}(F)$ of $F$ with respect to $\mathbb{P}_{\mathrm{SU}(N), \theta}$ is measurable with respect to $\theta$ and

$$
\int_{0}^{2 \pi} \mathbb{E}_{\mathrm{SU}(N), \theta}(F) \frac{\mathrm{d} \theta}{2 \pi}=\mathbb{E}_{U(N)}(F) .
$$

Proof. One has

$$
\mathbb{E}_{\mathrm{SU}(N), \theta}(F)=\int F\left(X \mathrm{e}^{\mathrm{i} \theta}\right) \mathrm{d} \mathbb{P}_{\mathrm{SU}(N)}(X),
$$

which, by dominated convergence, is continuous, and a fortiori measurable with respect to $\theta$. By integrating (3) with respect to $\theta$, one sees that the proposition is equivalent to the following: if $U$ is a uniform matrix on $\operatorname{SU}(N)$, and if $Z$ is independent, uniform on the 
unit circle, then $Z U$ is uniform on $U(N)$. Now, let $A$ be a deterministic matrix in $U(N)$. For any $d \in \mathbb{C}$ such that $d^{-N}=\operatorname{det}(A)$, one has $A d \in \operatorname{SU}(N)$, and then $Z U A=(Z / d)(U A d)$, where:

(1) $U$ Ad follows the Haar measure on $\mathrm{SU}(N)$ (since this measure is invariant by multiplication by $A d \in \mathrm{SU}(N))$.

(2) $Z / d$ is uniform on the unit circle (since $d$, as $\operatorname{det}(A)$, has modulus 1 ).

(3) These two variables, which depend deterministically on the independent variables $A$ and $Z$, are independent.

Hence, $Z U A$ has the same law as $Z U$, that is, this law is invariant by right multiplication by any unitary matrix. Hence, $Z U$ follows the Haar measure on $U(N)$.

Remark 2.2. This result also appears in the proof of [19, Lemma 2.5].

Remark 2.3. This disintegration will allow us to deduce the estimates on $\mathrm{SU}(N)$ we will need from the corresponding estimates on $U(N)$. Another way to proceed would be to make use of [13, Lemma AD.7.1] which we recall here.

Lemma 2.4 ([13, p. 390]). Let $f: U(N) \rightarrow \mathbb{C}$ be a $\mathcal{C}^{\infty}$ bounded central function, that is, $f(U V)=f(V U)$ for all $U, V \in U(N)$. Then, one has the equality with an absolutely convergent series

$$
\mathbb{E}_{\mathrm{SU}(N)}(f(X))=\sum_{\ell \in \mathbb{Z}} \mathbb{E}_{U(N)}\left((\operatorname{det} X)^{\ell} f(X)\right) .
$$

If one wished to proceed using this lemma, one would then need to estimate the terms of the series in order to show that the term corresponding to $\ell=0$ is dominant, that is, that the expectation $\mathbb{E}_{\mathrm{SU}(N)}(f(X))$ is close to $\mathbb{E}_{U(N)}(f(X))$. For $f$ being a function of the characteristic polynomial, this can be done using the probabilistic splitting defined in [4] and used in the proof of Lemma 3.2.

\subsection{Number of eigenvalues in an arc}

The result we state here relates the number of eigenvalues of a unitary matrix on a given arc to the logarithm of its characteristic polynomial. Recall that for $U \in U(N)$ and $\theta \in \mathbb{R}$, we denote by $Z_{U}(\theta)$ the characteristic polynomial of $U$ evaluated at $\mathrm{e}^{-\mathrm{i} \theta}$. Moreover, if $\mathrm{e}^{\mathrm{i} \theta}$ is not an eigenvalue of $U$, we define the logarithm of $Z_{U}(\theta)$, as

$$
\log Z_{U}(\theta):=\sum_{j=1}^{N} \log \left(1-\mathrm{e}^{\mathrm{i}\left(\theta_{j}-\theta\right)}\right)
$$


where $\theta_{1}, \ldots, \theta_{N}$ are the zeros of $Z_{U}$ in $[0,2 \pi)$, taken with multiplicity (note that the eigenvalues of $U$ are $\left.\mathrm{e}^{\mathrm{i} \theta_{1}}, \ldots, \mathrm{e}^{\mathrm{i} \theta_{N}}\right)$, and where the principal branch of the logarithm is taken in the right-hand side. Note that $\mathrm{e}^{\mathrm{i} \theta}$ is not an eigenvalue of $U$ almost surely under Haar measure on $U(N)$, and also almost surely under the Haar measure on $\mathrm{SU}(N)$, except for the case when $\mathrm{e}^{\mathrm{i} \theta}=1$ and $N=1$.

We then have the following result, already stated, for example, in [10].

Proposition 2.5. Let $0 \leqslant s<t<2 \pi$, and let us assume that $s$ and $t$ are not zeros of $Z_{U}$. Then, the number of zeros of $Z_{U}$ in the interval $(s, t)$ is given as follows:

$$
\sum_{k=1}^{N} \mathbb{1}_{\left\{\theta_{k} \in(s, t)\right\}}=\frac{N}{2 \pi}(t-s)+\frac{1}{\pi}\left(\operatorname{Im} \log Z_{U}(t)-\operatorname{Im} \log Z_{U}(s)\right) .
$$

Proof. It is sufficient to check that for all $\vartheta \in[0,2 \pi) \backslash\{s, t\}$,

$$
\pi \mathbb{1}_{\{\vartheta \in(s, t)\}}=\frac{t-s}{2}+\operatorname{Im} \log \left(1-\mathrm{e}^{\mathrm{i}(\vartheta-t)}\right)-\operatorname{Im} \log \left(1-\mathrm{e}^{\mathrm{i}(\vartheta-s)}\right) .
$$

Now, for $v \in(0,2 \pi)$,

$$
1-\mathrm{e}^{i v}=\mathrm{e}^{\mathrm{i} v / 2}\left(\mathrm{e}^{-\mathrm{i} v / 2}-\mathrm{e}^{\mathrm{i} v / 2}\right)=-2 \mathrm{i} \sin (v / 2) \mathrm{e}^{\mathrm{i} v / 2}=2 \sin (v / 2) \mathrm{e}^{\mathrm{i}(v-\pi) / 2} .
$$

Now, $\sin (v / 2)>0$ and $(v-\pi) / 2 \in(-\pi / 2, \pi / 2)$ and hence

$$
\operatorname{Im} \log \left(1-\mathrm{e}^{\mathrm{i} v}\right)=\frac{v-\pi}{2},
$$

since we take the principal branch of the logarithm. Now, for $\vartheta \in[0,2 \pi) \backslash\{s, t\}, \vartheta-s+$ $2 \pi \mathbb{1}_{\{\vartheta<s\}}$ and $\vartheta-t+2 \pi \mathbb{1}_{\{\vartheta<t\}}$ are in $(0,2 \pi)$, which implies

$$
\begin{aligned}
\operatorname{Im} \log \left(1-\mathrm{e}^{\mathrm{i}(\vartheta-t)}\right)-\operatorname{Im} \log \left(1-\mathrm{e}^{\mathrm{i}(\vartheta-s)}\right) & =\frac{\vartheta-t-\pi+2 \pi \mathbb{1}_{\{\vartheta<t\}}}{2}-\frac{\vartheta-s-\pi+2 \pi \mathbb{1}_{\{\vartheta<s\}}}{2} \\
& =\frac{s-t}{2}+\pi\left(\mathbb{1}_{\{\vartheta<t\}}-\mathbb{1}_{\{\vartheta<s\}}\right),
\end{aligned}
$$

from which Proposition 2.5 follows by summing over the $N$ zeros of $Z_{U}$ in $[0,2 \pi)$.

\section{Proof of Theorem 1.1}

\subsection{Conventions}

All the random matrices we will consider are defined, for some $N \geqslant 1$, on the measurable space $\left(\mathcal{M}_{N}(\mathbb{C}), \mathcal{F}\right)$, where $\mathcal{F}$ denotes the Borel $\sigma$-algebra of the space of $N \times N$ complex 
matrices $\mathcal{M}_{N}(\mathbb{C})$. The canonical random variable with values in $\mathcal{M}_{N}(\mathbb{C})$, that is, the identity function from $\left(\mathcal{M}_{N}(\mathbb{C}), \mathcal{F}\right)$ to $\mathcal{M}_{N}(\mathbb{C})$ is denoted by $X$. For example, if $F$ is a bounded, Borel function from $\mathcal{M}_{N}(\mathbb{C})$ to $\mathbb{R}$,

$$
\mathbb{E}_{\mathrm{SU}(N)}[F(X)]=\int_{\mathcal{M}_{N}(\mathbb{C})} F(M) d \mathbb{P}_{\mathrm{SU}(N)}(M) .
$$

\subsection{An estimate on the average of the logarithm of the characteristic polynomial}

Lemma 3.1. There exists a universal constant $c_{1}>0$ such that for all $N \geqslant 2$, and $A \geqslant 0$,

$$
\int_{0}^{2 \pi} \mathbb{P}_{\mathrm{SU}(N)}\left(\left|\log Z_{X}(\theta)\right| \geqslant A \sqrt{\log N)} \frac{\mathrm{d} \theta}{2 \pi} \leqslant c_{1} \mathrm{e}^{-\frac{A}{2}\left(A \wedge \frac{\sqrt{\log N}}{2}\right)},\right.
$$

where $A \wedge \frac{\sqrt{\log N}}{2}$ denotes the minimum of $A$ and $\frac{\sqrt{\log N}}{2}$.

Proof. For all $\lambda \geqslant 0$,

$$
\begin{aligned}
\int_{0}^{2 \pi} \mathbb{P}_{\mathrm{SU}(N)}\left(\left|\log Z_{X}(\theta)\right| \geqslant A \sqrt{\log N}\right) \frac{\mathrm{d} \theta}{2 \pi} & \leqslant \mathrm{e}^{-\lambda A \sqrt{\log N}} \int_{0}^{2 \pi} \mathbb{E}_{\mathrm{SU}(N)}\left(\mathrm{e}^{\lambda\left|\log Z_{X}(\theta)\right|}\right) \frac{\mathrm{d} \theta}{2 \pi} \\
& \leqslant \mathrm{e}^{-\lambda A \sqrt{\log N}} \mathbb{E}_{U(N)}\left(\mathrm{e}^{\lambda\left|\log Z_{X}(0)\right|}\right)(\mathrm{by}(2)) \\
& \leqslant \mathrm{e}^{-\lambda A \sqrt{\log N}} \mathbb{E}_{U(N)}\left(\mathrm{e}^{\lambda\left(\left|\operatorname{Re} \log Z_{X}(0)\right|+\left|\operatorname{Im} \log Z_{X}(0)\right|\right)}\right) .
\end{aligned}
$$

Using the inequality $\mathrm{e}^{|a|+|b|} \leqslant \mathrm{e}^{a+b}+\mathrm{e}^{a-b}+\mathrm{e}^{-a+b}+\mathrm{e}^{-a-b}$, valid for all $a, b \in \mathbb{R}$, and writing the right-hand side of this inequality as $4 \mathbb{E}\left(\mathrm{e}^{B a+B^{\prime} b}\right)$ for $B$ and $B^{\prime}$ two independent Bernoulli random variables, independent of $U$, such that $\mathbb{P}(B=1)=1-\mathbb{P}(B=-1)=\frac{1}{2}$, we have:

$$
\int_{0}^{2 \pi} \mathbb{P}_{\mathrm{SU}(N)}\left(\left|\log Z_{X}(\theta)\right| \geqslant A \sqrt{\log N}\right) \frac{\mathrm{d} \theta}{2 \pi} \leqslant 4 \mathrm{e}^{-\lambda A \sqrt{\log N}} \mathbb{E}_{U(N)}\left(\mathrm{e}^{\lambda\left(B \operatorname{Re} \log Z_{X}(0)+B^{\prime} \operatorname{Im} \log Z_{X}(0)\right)}\right) .
$$

Keating and Snaith [15] showed that for $s, t \in \mathbb{C}$ such that $\operatorname{Re}(s+\mathrm{i} t)$ and $\operatorname{Re}(s-\mathrm{i} t)$ are strictly larger than -1 ,

$$
\mathbb{E}_{U(N)}\left(\mathrm{e}^{s \operatorname{Re} \log Z_{X}(0)+t \operatorname{Im} \log Z_{X}(0)}\right)=\frac{G\left(1+\frac{s+\mathrm{i} t}{2}\right) G\left(1+\frac{s-\mathrm{i} t}{2}\right) G(1+N) G(1+N+s)}{G\left(1+N+\frac{s+\mathrm{i} t}{2}\right) G\left(1+N+\frac{s-\mathrm{i} t}{2}\right) G(1+s)},
$$

where $G$ is the Barnes $G$-function, defined for all $z \in \mathbb{C}$, by

$$
G(z+1):=(2 \pi)^{z / 2} \mathrm{e}^{-\left[(1+\gamma) z^{2}+z\right] / 2} \prod_{n=1}^{\infty}\left(1+\frac{z}{n}\right)^{n} \mathrm{e}^{-z+\left(z^{2} / 2 n\right)},
$$

with $\gamma$ being the Euler constant. 
12376 Y. Barhoumi-Andréani et al.

In other words, one has

$$
\mathbb{E}_{U(N)}\left(\mathrm{e}^{s \operatorname{Re} \log Z_{X}(0)+t \operatorname{Im} \log Z_{X}(0)}\right)=\frac{G\left(1+\frac{s+\mathrm{i} t}{2}\right) G\left(1+\frac{s-\mathrm{i} t}{2}\right)}{G(1+s)} N^{\left(s^{2}+t^{2}\right) / 4} G_{N, s, t},
$$

where, by the classical estimates of the Barnes function,

$$
G_{N, s, t}:=N^{-\left(s^{2}+t^{2}\right) / 4} \frac{G(1+N) G(1+N+s)}{G\left(1+N+\frac{s+\mathrm{i} t}{2}\right) G\left(1+N+\frac{s-\mathrm{i} t}{2}\right)}
$$

tends to 1 when $N$ goes to infinity, uniformly on $s$ and $t$ if these parameters are bounded.

For any sequence $\left(\lambda_{N}\right)_{N \geqslant 1}$ such that $\lambda_{N} \in\left[0, \frac{1}{2}\right]$, one has (taking $s=\lambda_{N} B$ and $\left.t=\lambda_{N} B^{\prime}\right)$ :

$$
\mathbb{E}_{U(N)}\left(\mathrm{e}^{\lambda_{N}\left(B \operatorname{Re} \log Z_{X}(0)+B^{\prime} \operatorname{Im} \log Z_{X}(0)\right)}\right)=M\left(\lambda_{N}\right) N^{\lambda_{N}^{2} / 2},
$$

where

$$
M\left(\lambda_{N}\right):=\mathbb{E}\left(\frac{G\left(1+\lambda_{N} \frac{B+\mathrm{i} B^{\prime}}{2}\right) G\left(1+\lambda_{N} \frac{B-\mathrm{i} B^{\prime}}{2}\right)}{G\left(1+\lambda_{N} B\right)} G_{N, \lambda_{N} B, \lambda_{N} B^{\prime}}\right) .
$$

Since the function $G$ is holomorphic, with no zero on the half-plane $\{z \in \mathbb{C}: \operatorname{Re}(z)>0\}$, and since $G_{N, \lambda B, \lambda B^{\prime}}$ tends to 1 when $N$ goes to infinity, uniformly on $\lambda \in\left[0, \frac{1}{2}\right]$, the quantity $M(\lambda)$ is uniformly bounded by some universal constant $C^{\prime}>0$, for $\lambda \in\left[0, \frac{1}{2}\right]$. Hence, for $N$ going to infinity,

$$
\mathbb{E}_{U(N)}\left(\mathrm{e}^{\lambda_{N}\left(B \operatorname{Re} \log Z_{X}(0)+B^{\prime} \operatorname{Im} \log Z_{X}(0)\right)}\right) \leqslant C^{\prime} N^{\lambda_{N}^{2} / 2},
$$

which implies that

$$
\int_{0}^{2 \pi} \mathbb{P}_{\mathrm{SU}(N)}\left(\left|\log Z_{X}(\theta)\right| \geqslant A \sqrt{\log N}\right) \frac{\mathrm{d} \theta}{2 \pi} \leqslant 4 C^{\prime} \mathrm{e}^{-\lambda_{N} A} \sqrt{\log N}+\left(\lambda_{N}^{2} \log N\right) / 2 .
$$

Now, taking $\lambda_{N}=(1 / 2) \wedge(A / \sqrt{\log N})$ (which means $\lambda_{N}$ is the smaller of $\frac{1}{2}$ and $\left.A / \sqrt{\log N}\right)$, we have

$$
\begin{aligned}
\int_{0}^{2 \pi} \mathbb{P}_{\mathrm{SU}(N)}\left(\left|\log Z_{X}(\theta)\right| \geqslant A \sqrt{\log N}\right) \frac{\mathrm{d} \theta}{2 \pi} & \leqslant 4 C^{\prime} \mathrm{e}^{-\lambda_{N} \sqrt{\log N}\left[A-\left(\lambda_{N} \sqrt{\log N}\right) / 2\right]} \\
& \leqslant 4 C^{\prime} \mathrm{e}^{-\lambda_{N} \sqrt{\log N}[A-(A / \sqrt{\log N})(\sqrt{\log N}) / 2]} \\
& \leqslant 4 C^{\prime} \mathrm{e}^{-\lambda_{N} \sqrt{\log N}(A / 2)} \\
& \leqslant 4 C^{\prime} \mathrm{e}^{-[(\sqrt{\log N} / 2) \wedge A](A / 2)} .
\end{aligned}
$$




\subsection{An estimate on the imaginary part of the logarithm of the characteristic polynomial}

From the previous result, we obtain the following estimate for the imaginary part of the logarithm of the characteristic polynomial.

Lemma 3.2. There exists a universal constant $c_{1}^{\prime}>0$ such that for all $N \geqslant 2, A \geqslant 0$, and $\theta \in \mathbb{R}$,

$$
\mathbb{P}_{\mathrm{SU}(N)}\left(\left|\operatorname{Im} \log Z_{X}(\theta)\right| \geqslant A \sqrt{\log N}\right) \leqslant C_{1}^{\prime} \mathrm{e}^{-\frac{A}{2}\left(A \wedge \frac{\sqrt{\log N}}{2}\right)} .
$$

Proof. We use here the probabilistic splitting established in [4] which shows that for any $U \in U(N)$, there exists, for $1 \leqslant j \leqslant N, x_{j}$ on the unit sphere of $\mathbb{C}^{j}$, uniquely determined, such that

$$
U=R\left(x_{N}\right)\left(\begin{array}{cc}
R\left(x_{N-1}\right) & 0 \\
0 & 1
\end{array}\right)\left(\begin{array}{cc}
R\left(x_{N-2}\right) & 0 \\
0 & I_{2}
\end{array}\right) \cdots\left(\begin{array}{cc}
R\left(x_{1}\right) & 0 \\
0 & I_{N-1}
\end{array}\right)
$$

where $R\left(x_{j}\right)$ denotes the unique unitary matrix in $U(j)$ sending the last basis vector $e_{j}$ of $\mathbb{C}^{j}$ to $x_{j}$, and such that the image of $I_{j}-R\left(x_{j}\right)$ is the vector space generated by $e_{j}-x_{j}$. (See also [5] for an infinite-dimensional point of view.)

Moreover, the characteristic polynomial of $U \in U(N)$ is given by

$$
Z_{U}(0)=\prod_{j=1}^{N}\left(1-\left\langle x_{j}, e_{j}\right\rangle\right)
$$

and its logarithm is

$$
\log Z_{U}(0)=\sum_{j=1}^{N} \log \left(1-\left\langle x_{j}, e_{j}\right\rangle\right)
$$

when 1 is not an eigenvalue of $U$, taking the principal branch of the logarithm on the right-hand side. Note that the determination of the logarithm given by this formula fits with the definition involving the eigenangles (4). Indeed, the two formulas depend continuously on the matrix $U$, on the connected set $\{U \in U(N), 1 \notin \operatorname{Spec}(U)\}$, and their exponentials are equal, hence, it is sufficient to check that they coincide for one matrix $U$. For example if $U=-I_{N}$, then $x_{j}=-e_{j}$ for all $j$ and the two formulas both give $N \log 2$.

If $U$ follows the uniform distribution on $U(N)$, then the vectors $\left(x_{j}\right)_{1 \leqslant j \leqslant N}$ are independent and $x_{j}$ is uniform on the sphere of $\mathbb{C}^{j}$. The determinant of $U$ is equal to the product of the determinants of $R\left(x_{j}\right)$ for $1 \leqslant j \leqslant N$, and since $R\left(x_{1}\right)$ is the multiplication 
by $x_{1}$ on $\mathbb{C}$, one has

$$
\operatorname{det}(U)=x_{1} \prod_{j=2}^{N} \Gamma_{j}\left(x_{j}\right)
$$

where $\Gamma_{j}$ is the function from $\mathbb{C}^{j}$ to the unit circle $\mathbb{U}$ that maps $x_{j} \mapsto \operatorname{det}\left(R\left(x_{j}\right)\right)$. From this, we deduce that under the measure $\mathbb{P}_{\mathrm{SU}(N), \theta}$

(1) The vectors $\left(x_{j}\right)_{2 \leqslant j \leqslant N}$ are independent, $x_{j}$ being uniform on the unit sphere of $\mathbb{C}^{j}$.

(2) The value of $x_{1} \in \mathbb{U}$ is uniquely determined by the determinant $\operatorname{det}(U)=\mathrm{e}^{\mathrm{i} N \theta}$,

$$
x_{1}=\mathrm{e}^{\mathrm{i} N \theta} \prod_{j=2}^{N}\left[\Gamma_{j}\left(x_{j}\right)\right]^{-1} .
$$

Indeed, let $\mathbb{P}_{\mathrm{SU}(N), \theta}^{\prime}$ be the probability measure on the image of $\mathrm{SU}(N)$ by the multiplication by $\mathrm{e}^{\mathrm{i} \theta}$, under which the law of $\left(x_{j}\right)_{1 \leqslant j \leqslant N}$ is given by the two bullet points above. This probability measure can be constructed as the law of the random matrix $U$ given by the formula (7), where $\left(x_{j}\right)_{1 \leqslant j \leqslant N}$ are random vectors whose joint distribution is given by the bullet points (1) and (2) just above. We now have to prove that $\mathbb{P}_{\mathrm{SU}(N), \theta}=\mathbb{P}_{\mathrm{SU}(N), \theta}^{\prime}$. Let us first note that the joint law of $\left(x_{j}\right)_{2 \leqslant j \leqslant N}$, under the probability measure $\mathbb{P}_{\mathrm{SU}(N), \theta}^{\prime}$, does not depend on $\theta$. Hence, under the averaged measure

$$
\int_{0}^{2 \pi} \mathbb{P}_{\mathrm{SU}(N), \theta}^{\prime} \frac{\mathrm{d} \theta}{2 \pi}
$$

the vectors $\left(x_{j}\right)_{2 \leqslant j \leqslant N}$ still have the same law, that is, they are independent and $x_{j}$ is uniform on the unit sphere of $\mathbb{C}^{j}$. Moreover, conditionally on $\left(x_{j}\right)_{2 \leqslant j \leqslant N}, x_{1}=$ $\mathrm{e}^{\mathrm{i} N \theta} \prod_{j=2}^{N}\left[\Gamma_{j}\left(x_{j}\right)\right]^{-1}$, where $\theta$ is uniform on $[0,2 \pi)$. Hence, $\left(x_{j}\right)_{1 \leqslant j \leqslant N}$ are independent, $x_{1}$ is uniform on $\mathbb{U}$, and thus $x_{j}$ in uniform on the unit sphere of $\mathbb{C}^{j}$ for all $j \in\{1, \ldots, N\}$, which implies

$$
\int_{0}^{2 \pi} \mathbb{P}_{\mathrm{SU}(N), \theta}^{\prime} \frac{\mathrm{d} \theta}{2 \pi}=\mathbb{P}_{U(N)}=\int_{0}^{2 \pi} \mathbb{P}_{\mathrm{SU}(N), \theta} \frac{\mathrm{d} \theta}{2 \pi} .
$$

Now, $\mathbb{P}_{\mathrm{SU}(N), 2 \pi / N}$ is the image of $\mathbb{P}_{\mathrm{SU}(N)}$ by multiplication by $\mathrm{e}^{\mathrm{i} 2 \pi / N} I_{N}$, which is a matrix in $\mathrm{SU}(N)$. The invariance property defining the Haar measure $\mathbb{P}_{\mathrm{SU}(N)}$ implies that $\mathbb{P}_{\mathrm{SU}(N), 2 \pi / N}=\mathbb{P}_{\mathrm{SU}(N)}$, and so $\theta \mapsto \mathbb{P}_{\mathrm{SU}(N), \theta}$ is $(2 \pi / N)$-periodic. It is the same for $\theta \mapsto \mathbb{P}_{\mathrm{SU}(N), \theta}^{\prime}$ since the values of $x_{1}, \ldots, x_{N}$ involved in the definition of $\mathbb{P}_{\mathrm{SU}(N), \theta}^{\prime}$ do not change if we add a multiple of $2 \pi / N$ to $\theta$. Hence,

$$
\int_{0}^{2 \pi / N} \mathbb{P}_{\mathrm{SU}(N), \theta}^{\prime} \frac{N \mathrm{~d} \theta}{2 \pi}=\int_{0}^{2 \pi / N} \mathbb{P}_{\mathrm{SU}(N), \theta} \frac{N \mathrm{~d} \theta}{2 \pi} .
$$


Now, let $F$ be a continuous, bounded function from $U(N)$ to $\mathbb{R}$. By applying the equality above to the function $U \mapsto F(U) \mathbb{1}_{\left\{\operatorname{det} U \in\left\{\mathrm{e}^{\mathrm{i} N \theta}, \theta \in I\right\}\right\}}$, for an interval $I \subset[0,2 \pi / N)$, one deduces with obvious notation that

$$
\int_{I} \mathbb{E}_{\mathrm{SU}(N), \theta}^{\prime}(F) \frac{\mathrm{d} \theta}{|I|}=\int_{I} \mathbb{E}_{\mathrm{SU}(N), \theta}(F) \frac{\mathrm{d} \theta}{|I|},
$$

where $|I|$ is the length of $I$. By the definition of $\mathbb{P}_{\mathrm{SU}(N), \theta}$ and $\mathbb{P}_{\mathrm{SU}(N), \theta}^{\prime}$, the first measure is the image of $\mathbb{P}_{\mathrm{SU}(N)}$ by multiplication by $\mathrm{e}^{\mathrm{i} \theta}$, and the second measure is the image of $\mathbb{P}_{\mathrm{SU}(N), 0}^{\prime}$ by right multiplication by the matrix $\left(\begin{array}{cc}\mathrm{e}^{\mathrm{i} N \theta} & 0 \\ 0 & I_{N-1}\end{array}\right)$. Hence, by continuity and boundedness of $F$, and by dominated convergence, $\mathbb{E}_{\mathrm{SU}(N), \theta}(F)$ and $\mathbb{E}_{\mathrm{SU}(N), \theta}^{\prime}(F)$ are continuous with respect to $\theta$. By considering a sequence $\left(I_{r}\right)_{r \geqslant 1}$ of intervals containing a given value of $\theta$ and whose length tends to zero, one deduces, by letting $r \rightarrow \infty$,

$$
\mathbb{E}_{\mathrm{SU}(N), \theta}^{\prime}(F)=\mathbb{E}_{\mathrm{SU}(N), \theta}(F)
$$

We now get the equality $\mathbb{P}_{\mathrm{SU}(N), \theta}=\mathbb{P}_{\mathrm{SU}(N), \theta}^{\prime}$, and thus the law of $\left(x_{j}\right)_{1 \leqslant j \leqslant N}$ under $\mathbb{P}_{\mathrm{SU}(N), \theta}$ described above.

Hence, the sequence $\left(x_{j}\right)_{2 \leqslant j \leqslant N}$ has the same law under $\mathbb{P}_{\mathrm{SU}(N), \theta}$ and $\mathbb{P}_{U(N)}$. We now use this fact to construct a coupling between these two probability measures on the unitary group.

The general principle of coupling is the following: When we want to show that two probability distributions $\mathbb{P}_{1}$ and $\mathbb{P}_{2}$ on a metric space have a similar behavior, a possible strategy is to construct a couple $\left(U, U^{\prime}\right)$ of random variables defined on the same probability space endowed with a probability $\mathbb{P}$, such that the law of $U$ under $\mathbb{P}$ is $\mathbb{P}_{1}$, the law of $U^{\prime}$ under $\mathbb{P}$ is $\mathbb{P}_{2}$, and the distance between $U$ and $U^{\prime}$ is small with high probability. In the present situation, we take $\left(x_{j}^{\prime}\right)_{1 \leqslant j \leqslant N}$ to be independent with $x_{j}^{\prime}$ uniform on the unit sphere of $\mathbb{C}^{j}$ for all $j \in\{1, \ldots, N\}$. We construct, using (7), a random matrix $U^{\prime}$ following $\mathbb{P}_{U(N)}$. Then, we do the coupling by taking $x_{j}:=x_{j}^{\prime}$ for $2 \leqslant j \leqslant N$ and

$$
X_{1}:=\mathrm{e}^{\mathrm{i} N \theta} \prod_{j=2}^{N}\left[\Gamma_{j}\left(x_{j}\right)\right]^{-1},
$$

which gives a random matrix $U$ following $\mathbb{P}_{\mathrm{SU}(N), \theta}$. From the fact that $x_{j}=x_{j}^{\prime}$ for $j \geqslant 2$ and equation (8), we get the following:

$$
\log Z_{U}(0)-\log Z_{U^{\prime}}(0)=\log \left(1-x_{1}\right)-\log \left(1-x_{1}^{\prime}\right)
$$


12380 Y. Barhoumi-Andréani et al.

and in particular,

$$
\left|\operatorname{Im} \log Z_{U}(0)-\operatorname{Im} \log Z_{U^{\prime}}(0)\right| \leqslant \pi .
$$

Now, for $B:=\left(A-\frac{\pi}{\sqrt{\log N}}\right)_{+}$, one gets:

$$
\begin{aligned}
\mathbb{P}_{\mathrm{SU}(N)}\left(\left|\operatorname{Im} \log Z_{X}(-\theta)\right| \geqslant A \sqrt{\log N}\right) & =\mathbb{P}_{\mathrm{SU}(N), \theta}\left(\left|\operatorname{Im} \log Z_{X}(0)\right| \geqslant A \sqrt{\log N}\right) \\
& =\mathbb{P}\left(\left|\operatorname{Im} \log Z_{U}(0)\right| \geqslant A \sqrt{\log N}\right) \\
& \leqslant \mathbb{P}\left(\left|\operatorname{Im} \log Z_{U^{\prime}}(0)\right| \geqslant A \sqrt{\log N}-\pi\right) \\
& =\mathbb{P}_{U(N)}\left(\left|\operatorname{Im} \log Z_{X}(0)\right| \geqslant B \sqrt{\log N}\right) \\
& =\int_{0}^{2 \pi} \mathbb{P}_{\mathrm{SU}(N)}\left(\left|\operatorname{Im} \log Z_{X}(\theta)\right| \geqslant B \sqrt{\log N}\right) \frac{\mathrm{d} \theta}{2 \pi} \\
& \leqslant \int_{0}^{2 \pi} \mathbb{P}_{\mathrm{SU}(N)}\left(\left|\log Z_{X}(\theta)\right| \geqslant B \sqrt{\log N}\right) \frac{\mathrm{d} \theta}{2 \pi} \\
& \leqslant C_{1} \mathrm{e}^{-\frac{B}{2}\left(B \wedge \frac{\sqrt{\log N}}{2}\right)} .
\end{aligned}
$$

Now, if $B \leqslant \frac{\sqrt{\log N}}{2}$, then

$$
\begin{aligned}
\frac{A}{2}\left(A \wedge \frac{\sqrt{\log N}}{2}\right) \leqslant \frac{A^{2}}{2} & \leqslant \frac{1}{2}\left(B+\frac{\pi}{\sqrt{\log N}}\right)^{2}=\frac{B^{2}}{2}+\frac{B \pi}{\sqrt{\log N}}+\frac{\pi^{2}}{2 \log N} \\
& \leqslant \frac{B^{2}}{2}+\frac{\pi}{2}+\frac{\pi^{2}}{2 \log 2} \\
& =\frac{B}{2}\left(B \wedge \frac{\sqrt{\log N}}{2}\right)+\frac{\pi}{2}+\frac{\pi^{2}}{2 \log 2} .
\end{aligned}
$$

If $B \geqslant \frac{\sqrt{\log N}}{2}$, then

$$
\begin{aligned}
\frac{A}{2}\left(A \wedge \frac{\sqrt{\log N}}{2}\right) \leqslant \frac{A \sqrt{\log N}}{4} & \leqslant \frac{\sqrt{\log N}}{4}\left(B+\frac{\pi}{\sqrt{\log N}}\right)=\frac{B \sqrt{\log N}}{4}+\frac{\pi}{4} \\
& =\frac{B}{2}\left(B \wedge \frac{\sqrt{\log N}}{2}\right)+\frac{\pi}{4}
\end{aligned}
$$

Hence, we get Lemma 3.2, with

$$
c_{1}^{\prime}=C_{1} \mathrm{e}^{\frac{\pi}{2}+\frac{\pi^{2}}{2 \log 2}} .
$$




\subsection{Bound on the concentration of the law of the logarithm of the characteristic polynomial}

Lemma 3.3. For $N \geqslant 4, \theta \in[0,2 \pi)$ and $\delta \in(0,1 / 2)$, one has, uniformly in $x_{0} \in \mathbb{R}$

$$
\mathbb{P}_{\mathrm{SU}(N)}\left[|\log | Z_{X}(\theta)\left|-x_{0}\right| \leqslant \delta \sqrt{\log N}\right] \leqslant C \delta \log (1 / \delta)
$$

where $C>0$ is a universal constant.

Proof. The proof of Lemma 3.3 needs several steps.

Sublemma 3.4. For $j \geqslant 1$ integer, $s, t \in \mathbb{R}$, let us define

$$
Q(j, s, t):=\frac{\left(j+\frac{\mathrm{i} t-s}{2}\right)\left(j+\frac{\mathrm{i} t+s}{2}\right)}{j(j+\mathrm{i} t)}
$$

Then,

(1) For $s^{2}+t^{2} \geqslant 8 j^{2},|Q(j, s, t)| \geqslant \max \left(1, \frac{\sqrt{s^{2}+t^{2}}}{8 j}\right)$.

(2) For $j^{2} \leqslant s^{2}+t^{2} \leqslant 8 j^{2},|Q(j, s, t)| \leqslant 1$.

(3) For $s^{2}+t^{2} \leqslant j^{2},|Q(j, s, t)| \leqslant \mathrm{e}^{-\left(s^{2}+t^{2}\right) / 10 j^{2}}$.

Proof. One has

$$
Q(j, s, t)=\frac{1-\frac{s^{2}+t^{2}}{4 j^{2}}+\mathrm{i} t / j}{1+\mathrm{i} t / j}
$$

If $s^{2}+t^{2} \leqslant 8 j^{2}$, it is immediate that the numerator has a smaller absolute value than the denominator, that is, $|Q(j, s, t)| \leqslant 1$. Moreover,

$$
|Q(j, s, t)|^{2}=\frac{1-\frac{s^{2}+t^{2}}{2 j^{2}}+\frac{\left(s^{2}+t^{2}\right)^{2}}{16 j^{4}}+\frac{t^{2}}{j^{2}}}{1+\frac{t^{2}}{j^{2}}}=1-\frac{\left(\frac{s^{2}+t^{2}}{2 j^{2}}\right)\left(1-\frac{s^{2}+t^{2}}{8 j^{2}}\right)}{1+\frac{t^{2}}{j^{2}}}
$$

and in the case where $s^{2}+t^{2} \leqslant j^{2}$, one deduces

$$
|Q(j, s, t)|^{2} \leqslant 1-\frac{7\left(s^{2}+t^{2}\right)}{32 j^{2}}
$$

and then

$$
|Q(j, s, t)| \leqslant \mathrm{e}^{-7\left(s^{2}+t^{2}\right) / 64 j^{2}} \leqslant \mathrm{e}^{-\left(s^{2}+t^{2}\right) / 10 j^{2}} .
$$


Now, if $s^{2}+t^{2} \geqslant 8 j^{2}$, the numerator in (9) has a larger absolute value than the denominator, and then $|Q(j, s, t)| \geqslant 1$. Moreover, since $\left(s^{2}+t^{2}\right) / 8 j^{2} \geqslant 1$,

$$
\begin{aligned}
|Q(j, s, t)|^{2} & =\frac{\left(\frac{s^{2}+t^{2}}{4 j^{2}}-1\right)^{2}+\frac{t^{2}}{j^{2}}}{1+\frac{t^{2}}{j^{2}}} \geqslant \frac{\left(\frac{s^{2}+t^{2}}{8 j^{2}}\right)^{2}+\frac{t^{2}}{j^{2}}}{1+\frac{t^{2}}{j^{2}}} \geqslant \frac{\left(\frac{s^{2}+t^{2}}{8 j^{2}}\right)^{2}+\frac{s^{2}+t^{2}}{j^{2}}}{1+\frac{s^{2}+t^{2}}{j^{2}}} \\
& \geqslant \frac{1}{64} \cdot \frac{\left(\frac{s^{2}+t^{2}}{j^{2}}\right)^{2}+\frac{s^{2}+t^{2}}{j^{2}}}{1+\frac{s^{2}+t^{2}}{j^{2}}}=\frac{s^{2}+t^{2}}{64 j^{2}},
\end{aligned}
$$

which finishes the proof of the sublemma.

Sublemma 3.5. Let $j \geqslant 1$ be an integer, let $\rho_{j}$ and $\sigma_{j}$ be the real and imaginary parts of $\log \left(1-\sqrt{\beta_{1, j-1}} \mathrm{e}^{\mathrm{i} \theta}\right)$, where $\beta_{1, j-1}$ is a beta random variable with $\beta(1, j-1)$ distribution and $\theta$ is independent of $\beta_{1, j-1}$, uniform on $[0,2 \pi]$. Then, for $s, t \in \mathbb{R}$,

$$
\left|\mathbb{E}\left[\mathrm{e}^{\mathrm{i}\left(t \rho_{j}+s \sigma_{j}\right)}\right]\right| \leqslant \mathrm{e}^{-\left(s^{2}+t^{2}\right) / 30 j}
$$

if $s^{2}+t^{2} \leqslant 8 j^{2}$, and

$$
\left|\mathbb{E}\left[\mathrm{e}^{\mathrm{i}\left(t \rho_{j}+s \sigma_{j}\right)}\right]\right| \leqslant \frac{8}{\sqrt{s^{2}+t^{2}}}
$$

if $s^{2}+t^{2} \geqslant 8 j^{2}$ and $j \geqslant 2$.

Proof. For $t \in \mathbb{R}$ and $s \in \mathbb{C}$ with real part strictly between -1 and 1 ,

$$
\mathbb{E}\left[\mathrm{e}^{\mathrm{i}\left(t \rho_{j}+s \sigma_{j}\right)}\right]=\frac{\Gamma(j) \Gamma(j+\mathrm{i} t)}{\Gamma\left(j+\frac{\mathrm{i} t-s}{2}\right) \Gamma\left(j+\frac{\mathrm{i} t+s}{2}\right)}
$$

(see [4]). Now, if $t$ is fixed, the function

$$
s \mapsto \mathbb{E}\left[\mathrm{e}^{\mathrm{i}\left(t \rho_{j}+s \sigma_{j}\right)}\right]
$$

is holomorphic, since the imaginary part is uniformly bounded (by $\pi / 2$ ). This implies that (10) holds for all $t \in \mathbb{R}, s \in \mathbb{C}$, and in particular for all $s, t \in \mathbb{R}$. Moreover,

$$
\frac{\Gamma(k) \Gamma(k+\mathrm{i} t)}{\Gamma\left(k+\frac{\mathrm{i} t-s}{2}\right) \Gamma\left(k+\frac{\mathrm{i} t+s}{2}\right)} \underset{k \rightarrow \infty}{\longrightarrow} 1,
$$

since $\Gamma(k+z) / \Gamma(k)$ is asymptotic to $k^{z}$ for large $k$ for all $z \in \mathbb{C}$. Hence, by using the equation $\Gamma(z+1)=z \Gamma(z)$, one deduces that

$$
\mathbb{E}\left[\mathrm{e}^{\mathrm{i}\left(t_{\rho}+s \sigma_{j}\right)}\right]=\prod_{k=j}^{\infty} \frac{\left(k+\frac{\mathrm{i} t-s}{2}\right)\left(k+\frac{\mathrm{i} t+s}{2}\right)}{k(k+\mathrm{i} t)}=\prod_{k=j}^{\infty} Q(k, s, t) .
$$


If $s^{2}+t^{2} \leqslant 8 j^{2}$, then $|Q(k, s, t)| \leqslant 1$ for all $k \geqslant j$ and $|Q(k, s, t)| \leqslant \mathrm{e}^{-\left(s^{2}+t^{2}\right) / 10 k^{2}}$ for all $k \geqslant 3 j$. Hence,

$$
\left|\mathbb{E}\left[\mathrm{e}^{\mathrm{i}\left(t \rho_{j}+s \sigma_{j}\right)}\right]\right| \leqslant \prod_{k=3 j}^{\infty} \mathrm{e}^{-\left(s^{2}+t^{2}\right) / 10 k^{2}} \leqslant \prod_{k=3 j}^{\infty} \mathrm{e}^{-\left(s^{2}+t^{2}\right) / 10 k(k+1)}=\mathrm{e}^{-\left(s^{2}+t^{2}\right) / 30 j} .
$$

Now let us assume $s^{2}+t^{2} \geqslant 8 j^{2}$. One has:

$$
\mathbb{E}\left[\mathrm{e}^{\mathrm{i}\left(t \rho_{j}+s \sigma_{j}\right)}\right]=\frac{\Gamma(1) \Gamma(1+\mathrm{i} t)}{\Gamma\left(1+\frac{\mathrm{i} t-s}{2}\right) \Gamma\left(1+\frac{\mathrm{i} t+s}{2}\right)} \prod_{k=1}^{j-1} \frac{1}{Q(k, s, t)},
$$

where all the factors $\frac{1}{Q(k, s, t)}$ have absolute value bounded by one. By considering the case where $j=1$, one deduces

$$
\left|\frac{\Gamma(1) \Gamma(1+\mathrm{i} t)}{\Gamma\left(1+\frac{\mathrm{i} t-s}{2}\right) \Gamma\left(1+\frac{\mathrm{i} t+s}{2}\right)}\right| \leqslant 1,
$$

and then, for $j \geqslant 2$,

$$
\left|\mathbb{E}\left[\mathrm{e}^{\mathrm{i}\left(t t_{j}+s \sigma_{j}\right)}\right]\right| \leqslant \frac{1}{|Q(1, s, t)|} \leqslant \frac{8}{\sqrt{s^{2}+t^{2}}} .
$$

Sublemma 3.6. For $N \geqslant 4$ and $\theta \in[0,2 \pi)$, the distribution of $\log \left(Z_{X}(\theta)\right)$ under Haar measure on $U(N)$ has a density with respect to Lebesgue measure on $\mathbb{C}$, which is continuous and bounded by $C_{0} / \log (N)$, where $C_{0}>0$ is a universal constant.

Proof. By the results in [4] and the previous sublemma, one checks that the characteristic function $\Phi$ of $\log \left(Z_{X}(\theta)\right) \in \mathbb{C} \sim \mathbb{R}^{2}$ is given by

$$
\Phi(s, t)=\prod_{j=1}^{N} \mathbb{E}\left[\mathrm{e}^{\mathrm{i}\left(t \rho_{j}+s \sigma_{j}\right)}\right] .
$$

If $s^{2}+t^{2} \geqslant 32 N$, one has $s^{2}+t^{2} \geqslant 128 \geqslant 8 j^{2}$ for $j \in\{2,3,4\}$. Hence,

$$
|\Phi(s, t)| \leqslant\left|\mathbb{E}\left[\mathrm{e}^{\mathrm{i}\left(t \rho_{2}+s \sigma_{2}\right)}\right]\right|\left|\mathbb{E}\left[\mathrm{e}^{\mathrm{i}\left(t \rho_{3}+s \sigma_{3}\right)}\right]\right|\left|\mathbb{E}\left[\mathrm{e}^{\mathrm{i}\left(t \rho_{4}+s \sigma_{4}\right)}\right]\right| \leqslant \frac{512}{\left(s^{2}+t^{2}\right)^{3 / 2}} .
$$

If $s^{2}+t^{2} \leqslant 32 N$, then $s^{2}+t^{2} \leqslant 8 j^{2}$ for all $j \geqslant 2 \sqrt{N}$. Hence,

$$
|\Phi(s, t)| \leqslant \prod_{2 \sqrt{N} \leqslant j \leqslant N} \mathbb{E}\left[\mathrm{e}^{\mathrm{i}\left(t p_{j}+s \sigma_{j}\right)}\right] \leqslant \exp \left(-\left(s^{2}+t^{2}\right) \sum_{2 \sqrt{N} \leqslant j \leqslant N} \frac{1}{30 j}\right) .
$$


12384 Y. Barhoumi-Andréani et al.

Since $\mathrm{e}^{1 / j} \geqslant \frac{j+1}{j}$, one deduces

$$
\begin{aligned}
|\Phi(s, t)| & \leqslant \prod_{2 \sqrt{N} \leqslant j \leqslant N}\left(\frac{j}{j+1}\right)^{\left(s^{2}+t^{2}\right) / 30} \leqslant\left(\frac{2 \sqrt{N}+1}{N+1}\right)^{\left(s^{2}+t^{2}\right) / 30} \\
& \leqslant\left(\frac{3 \sqrt{N}}{N}\right)^{\left(s^{2}+t^{2}\right) / 30}=\mathrm{e}^{-\log (N / 9)\left(s^{2}+t^{2}\right) / 60}
\end{aligned}
$$

Now, for $N \geqslant 10$,

$$
\begin{aligned}
\int_{\mathbb{R}^{2}}|\Phi(s, t)| \mathrm{d} s \mathrm{~d} t & \leqslant \int_{\mathbb{R}^{2}} \frac{512}{\left(s^{2}+t^{2}\right)^{3 / 2}} \mathbb{1}_{\left\{s^{2}+t^{2} \geqslant 32 N\right\}} \mathrm{d} s \mathrm{~d} t+\int_{\mathbb{R}^{2}} \mathrm{e}^{-\log (N / 9)\left(s^{2}+t^{2}\right) / 60} \mathbb{1}_{\left\{s^{2}+t^{2} \leqslant 32 N\right\}} \mathrm{d} s \mathrm{~d} t \\
& =\pi\left(\int_{0}^{32 N} \mathrm{e}^{-u \log (N / 9) / 60} \mathrm{~d} u+\int_{32 N}^{\infty} \frac{512}{u^{-3 / 2}} \mathrm{~d} u\right) \\
& \leqslant \frac{60 \pi}{\log (N / 9)}+1024 \pi(32 N)^{-1 / 2} \leqslant \frac{10,000}{\log N}
\end{aligned}
$$

and for $N \in\{4,5,6,7,8,9\}$,

$$
\begin{aligned}
\int_{\mathbb{R}^{2}}|\Phi(s, t)| \mathrm{d} s \mathrm{~d} t & \leqslant \int_{\mathbb{R}^{2}} \frac{512}{\left(s^{2}+t^{2}\right)^{3 / 2}} 1_{\left\{s^{2}+t^{2} \geqslant 32 N\right\}} \mathrm{d} s \mathrm{~d} t+\int_{\mathbb{R}^{2}} 1_{\left\{s^{2}+t^{2} \leqslant 32 N\right\}} \mathrm{d} s \mathrm{~d} t \\
& =\pi\left(\int_{0}^{32 N} \mathrm{~d} u+\int_{32 N}^{\infty} \frac{512}{u^{-3 / 2}} \mathrm{~d} u\right) \\
& \leqslant 32 \pi N+1024 \pi(32 N)^{-1 / 2} \leqslant 288 \pi+1024 \pi(128)^{-1 / 2} \leqslant \frac{10,000}{\log 9} .
\end{aligned}
$$

By applying Fourier inversion, we obtain Sublemma 3.6.

Let us now go back to the proof of Lemma 3.3. For any $X \in U(N)$ with eigenvalues $\left(\mathrm{e}^{\mathrm{i} \theta_{j}}\right)_{1 \leqslant j \leqslant N}$, one has, in the case where $\mathrm{e}^{\mathrm{i} \theta} \neq \mathrm{e}^{\mathrm{i} \theta_{j}}$ for all $j \in\{1, \ldots, N\}$,

$$
\begin{aligned}
\mathcal{I}:=\operatorname{Im}\left(\log \left(Z_{X}(\theta)\right)\right. & =\sum_{1 \leqslant j \leqslant N} \operatorname{Im}\left(\log \left(1-\mathrm{e}^{\mathrm{i}\left(\theta_{j}-\theta\right)}\right)\right) \\
& =\frac{1}{2} \sum_{1 \leqslant j \leqslant N}\left(\theta_{j}-\theta\right)+\sum_{1 \leqslant j \leqslant N} \operatorname{Im}\left(\log \left(\mathrm{e}^{-\mathrm{i}\left(\theta_{j}-\theta\right) / 2}-\mathrm{e}^{\mathrm{i}\left(\theta_{j}-\theta\right) / 2}\right)\right) \\
& \left.=\frac{1}{2} \operatorname{Im}(\log \operatorname{det}(X))-\frac{N \theta}{2}+\sum_{1 \leqslant j \leqslant N} \operatorname{Im}\left(\log \left(-2 \mathrm{i} \sin \left(\theta_{j}-\theta\right) / 2\right)\right)\right) \\
& =\frac{\mathcal{J}}{2}-\frac{N(\theta+\pi)}{2},
\end{aligned}
$$


where $\mathcal{J}$ denotes the version of $\operatorname{Im}(\log \operatorname{det}(X))$ lying on the interval $(-\pi, \pi]$. Hence, for any $\epsilon \in(0, \pi),|\mathcal{J}| \leqslant \epsilon$ if and only if $\mathcal{I}$ is on an interval of the form $\left[\frac{2 k \pi-\epsilon-N(\theta+\pi)}{2}, \frac{2 k \pi+\epsilon-N(\theta+\pi)}{2}\right]$ for some $k \in \mathbb{Z}$. Now, for some $A>0$ chosen later as a function of $\delta$, let $\Phi$ be a continuous function from $\mathbb{C}$ to $[0,1]$ such that $\Phi(z)=1$ if $\mid \operatorname{Re}$ $z-x_{0} \mid \leqslant \delta \sqrt{\log N}$ and $|\operatorname{Im} z| \leqslant A \sqrt{\log N}$, and such that $\Phi(z)=0$ for $\left|\operatorname{Re} z-x_{0}\right| \geqslant 2 \delta \sqrt{\log N}$ or $|\operatorname{Im} z| \geqslant 2 A \sqrt{\log N}$. For $\epsilon \in(0, \pi)$, and under the Haar measure $\mathbb{P}_{U(N)}$ on $U(N)$,

$$
\begin{aligned}
& \frac{\pi}{\epsilon} \mathbb{E}_{U(N)}\left[\Phi\left(\log \left(Z_{X}(\theta)\right)\right) \mathbb{1}_{\{|\mathcal{J}| \leqslant \epsilon\}}\right] \\
& \quad=\frac{\pi}{\epsilon} \sum_{k \in \mathbb{Z}} \mathbb{E}_{U(N)}\left[\Phi\left(\log \left(Z_{X}(\theta)\right)\right) \mathbb{1}_{\left\{\frac{2 k \pi-\epsilon-N(\theta+\pi)}{2} \leqslant \mathcal{I} \leqslant \frac{2 k \pi+\epsilon-N(\theta+\pi)}{2}\right\}}\right] \\
& \quad=\frac{\pi}{\epsilon} \sum_{k \in \mathbb{Z}} \int_{-\infty}^{\infty} \mathrm{d} x \int_{(2 k \pi-\epsilon-N(\theta+\pi)) / 2}^{(2 k \pi+\epsilon-N(\theta+\pi)) / 2} \mathrm{~d} y D(x+\mathrm{i} y) \Phi(x+\mathrm{i} y) \\
& \quad=\pi \sum_{k \in \mathbb{Z}} \int_{-\infty}^{\infty} \mathrm{d} x \int_{-1 / 2}^{1 / 2} \mathrm{~d} u D(x+\mathrm{i}[k \pi-N(\theta+\pi) / 2+u \epsilon]) \Phi(x+\mathrm{i}[k \pi-N(\theta+\pi) / 2+u \epsilon]),
\end{aligned}
$$

where $D$ denotes the density of the law of $\log \left(Z_{X}(\theta)\right)$, with respect to the Lebesgue measure. Now,

$$
D(x+\mathrm{i}[k \pi-N(\theta+\pi) / 2+u \epsilon]) \Phi(x+\mathrm{i}[k \pi-N(\theta+\pi) / 2+u \epsilon])
$$

is uniformly bounded by the overall maximum of $D$ and vanishes as soon as $\left|x-x_{0}\right| \geqslant$ $2 \delta \sqrt{\log N}$ or $|k| \pi \geqslant N(|\theta|+\pi) / 2+\pi / 2+2 A \sqrt{\log N}$. Since $D$ and $\Phi$ are continuous functions, one can apply dominated convergence and deduce that

$$
\frac{\pi}{\epsilon} \mathbb{E}_{U(N)}\left[\Phi\left(\log \left(Z_{X}(\theta)\right)\right) \mathbb{1}_{\{|\mathcal{J}| \leqslant \epsilon\}}\right]
$$

converges to

$$
\pi \sum_{k \in \mathbb{Z}} \int_{-\infty}^{\infty} D(x+\mathrm{i}[k \pi-N(\theta+\pi) / 2]) \Phi(x+\mathrm{i}[k \pi-N(\theta+\pi) / 2]) \mathrm{d} x
$$

when $\epsilon$ goes to zero. On the other hand, if the matrix $X$ follows $\mathbb{P}_{\mathrm{SU}(N)}$ and if $T$ is an independent uniform variable on $(-\pi, \pi]$, then $X \mathrm{e}^{\mathrm{i} T / N}$ follows $\mathbb{P}_{U(N)}$ and its determinant is $\mathrm{e}^{\mathrm{i} T}$. One deduces

$$
\begin{aligned}
& \frac{\pi}{\epsilon} \mathbb{E}_{U(N)}\left[\Phi\left(\log \left(Z_{X}(\theta)\right)\right) \mathbb{1}_{\{|\mathcal{J}| \leqslant \epsilon\}}\right]=\frac{\pi}{\epsilon} \mathbb{E}_{\mathrm{SU}(N)}\left[\Phi\left(\log \left(Z_{X \mathrm{X}^{\mathrm{i} T / N}}(\theta)\right)\right) \mathbb{1}_{\{|T| \leqslant \epsilon\}}\right] \\
& =\frac{1}{2 \epsilon} \int_{-\epsilon}^{\epsilon} \mathbb{E}_{\mathrm{SU}(N)}\left[\Phi\left(\log \left(Z_{\mathrm{Xe}}^{\mathrm{i} t / N}(\theta)\right)\right)\right] \mathrm{d} t
\end{aligned}
$$

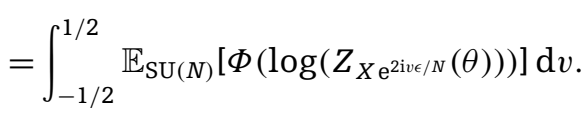


Now, the function $X \mapsto \Phi\left(\log \left(Z_{X}(\theta)\right)\right)$ is continuous from $U(N)$ to [0,1], since $\Phi$ is continuous with compact support and $X \mapsto \log \left(Z_{X}(\theta)\right)$ has discontinuities only at points where its real part goes to $-\infty$. One can then apply dominated convergence and obtain

$$
\frac{\pi}{\epsilon} \mathbb{E}_{U(N)}\left[\Phi\left(\log \left(Z_{X}(\theta)\right)\right) \mathbb{1}_{\{|\mathcal{J}| \leqslant \epsilon\}}\right] \underset{\epsilon \rightarrow 0}{\longrightarrow} \mathbb{E}_{\mathrm{SU}(N)}\left[\Phi\left(\log \left(Z_{X}(\theta)\right)\right)\right]
$$

By comparing with the convergence obtained just above, one deduces that

$$
\mathbb{E}_{\mathrm{SU}(N)}\left[\Phi\left(\log \left(Z_{X}(\theta)\right)\right)\right]=\pi \sum_{k \in \mathbb{Z}} \int_{-\infty}^{\infty} D(x+\mathrm{i}[k \pi-N(\theta+\pi) / 2]) \Phi(x+\mathrm{i}[k \pi-N(\theta+\pi) / 2]) \mathrm{d} x
$$

Since $D(z) \leqslant C_{0} / \log N$ and

$$
\mathbb{1}_{\left\{\left|x-x_{0}\right| \leqslant \delta \sqrt{\log N},|y| \leqslant A \sqrt{\log N}\right\}} \leqslant \Phi(x+\mathrm{i} y) \leqslant \mathbb{1}_{\left\{\left|x-x_{0}\right| \leqslant 2 \delta \sqrt{\log N},|y| \leqslant 2 A \sqrt{\log N}\right\}}
$$

for all $x, y \in \mathbb{R}$, one deduces

$$
\mathbb{P}_{\mathrm{SU}(N)}\left[|\log | Z_{X}(\theta)\left|-x_{0}\right| \leqslant \delta \sqrt{\log N},\left|\operatorname{Im} \log Z_{X}(\theta)\right| \leqslant A \sqrt{\log N}\right] \leqslant \frac{\pi d L C_{0}}{\log N},
$$

where $d=4 \delta \sqrt{\log N}$ is the length of the interval $\left[x_{0}-2 \delta \sqrt{\log N}, x_{0}+2 \delta \sqrt{\log N}\right]$ and $L$ is the number of integers $k$ such that $|k \pi-N(\theta+\pi) / 2| \leqslant 2 A \sqrt{\log N}$. Now, it is easy to check that $L \leqslant 1+\frac{4 A \sqrt{\log N}}{\pi}$, and so

$$
\mathbb{P}_{\mathrm{SU}(N)}\left[|\log | Z_{X}(\theta)\left|-X_{0}\right| \leqslant \delta \sqrt{\log N},\left|\operatorname{Im} \log Z_{X}(\theta)\right| \leqslant A \sqrt{\log N}\right] \leqslant 16 C_{0} A \delta+\frac{4 \pi \delta C_{0}}{\sqrt{\log N}}
$$

Using Lemma 3.2, one obtains

$$
\mathbb{P}_{\mathrm{SU}(N)}\left[|\log | Z_{X}(\theta)\left|-x_{0}\right| \leqslant \delta \sqrt{\log N}\right] \leqslant 16 C_{0} A \delta+\frac{4 \pi \delta C_{0}}{\sqrt{\log N}}+C_{1}^{\prime} \mathrm{e}^{-\frac{A}{2}\left(A \wedge \frac{\sqrt{\log N}}{2}\right)} .
$$

Let us now choose $A:=1+5 \log (1 / \delta)$. One finds

$$
A \wedge \frac{\sqrt{\log N}}{2}=[1+5 \log (1 / \delta)] \wedge \frac{\sqrt{\log N}}{2} \geqslant \frac{\sqrt{\log 2}}{2}
$$

and thus

$$
\frac{A}{2}\left(A \wedge \frac{\sqrt{\log N}}{2}\right) \geqslant \frac{5 \sqrt{\log 2} \log (1 / \delta)}{4} \geqslant \log (1 / \delta)
$$

Therefore,

$$
\mathbb{P}_{\mathrm{SU}(N)}\left[|\log | Z_{X}(\theta)\left|-x_{0}\right| \leqslant \delta \sqrt{\log N}\right] \leqslant 16 C_{0} \delta+80 C_{0} \delta \log (1 / \delta)+\frac{4 \pi \delta C_{0}}{\sqrt{\log N}}+C_{1}^{\prime} \delta .
$$


Since $\delta<\frac{1}{2}$, one has $\delta \leqslant \delta \log (1 / \delta) / \log (2)$, which implies Lemma 3.3, for

$$
C=\frac{16 C_{0}}{\log 2}+80 C_{0}+\frac{4 \pi C_{0}}{(\log 2)^{3 / 2}}+\frac{C_{1}^{\prime}}{\log 2} .
$$

\subsection{Behavior of the oscillation in short intervals of the logarithm of the characteristic polynomial}

Lemma 3.7. There exists $C_{2}>0$ such that for $\mu \in \mathbb{R}$ and $A \geqslant 0$ and uniformly in $N \geqslant M \geqslant$ $2 \vee \frac{|\mu|}{2 \pi}$

$$
\mathbb{P}_{\mathrm{SU}(N)}\left(\int_{0}^{2 \pi}\left|\operatorname{Re} \log Z_{X}\left(\theta+\frac{\mu}{N}\right)-\operatorname{Re} \log Z_{X}(\theta)\right| \frac{\mathrm{d} \theta}{2 \pi} \geqslant A \sqrt{\log M}\right) \leqslant \frac{C_{2}}{A^{2}}
$$

and

$$
\mathbb{P}_{\mathrm{SU}(N)}\left(\int_{0}^{2 \pi}\left|\operatorname{Im} \log Z_{X}\left(\theta+\frac{\mu}{N}\right)-\operatorname{Im} \log Z_{X}(\theta)\right| \frac{\mathrm{d} \theta}{2 \pi} \geqslant A \sqrt{\log M}\right) \leqslant \frac{C_{2}}{A^{2}} .
$$

Proof. By symmetry of the problem, we can assume $\mu>0$. Setting

$$
R_{\theta}:=\operatorname{Re} \log Z_{X}\left(\theta+\frac{\mu}{N}\right)-\operatorname{Re} \log Z_{X}(\theta)
$$

for fixed $\mu$ (or the same expression with the imaginary part), we get:

$$
\begin{aligned}
\mathbb{P}_{\mathrm{SU}(N)}\left(\int_{0}^{2 \pi}\left|R_{\theta}\right| \frac{\mathrm{d} \theta}{2 \pi} \geqslant A \sqrt{\log M}\right) & \leqslant \frac{1}{A^{2} \log M} \mathbb{E}_{\mathrm{SU}(N)}\left(\left(\int_{0}^{2 \pi}\left|R_{\theta}\right| \frac{\mathrm{d} \theta}{2 \pi}\right)^{2}\right) \\
& \leqslant \frac{1}{A^{2} \log M} \mathbb{E}_{\mathrm{SU}(N)}\left(\int_{0}^{2 \pi} R_{\theta}^{2} \frac{\mathrm{d} \theta}{2 \pi}\right) \\
& =\frac{1}{A^{2} \log M} \int_{0}^{2 \pi} \mathbb{E}_{\mathrm{SU}(N)}\left(R_{\theta}^{2}\right) \frac{\mathrm{d} \theta}{2 \pi} \\
& =\frac{1}{A^{2} \log M} \mathbb{E}_{U(N)}\left(R_{0}^{2}\right) \quad(\text { by }(2)) .
\end{aligned}
$$

Now, under $U(N)$, the canonical matrix $X$ is almost surely unitary. Let $\theta_{1}, \ldots, \theta_{N}$ be its eigenangles in $[0,2 \pi)$. For $j \in\{1, \ldots, N\}$ and $t \in[0,2 \pi) \backslash\left\{\theta_{j}\right\}$, we can expand the logarithm as a conditionally convergent series

$$
\log \left(1-\mathrm{e}^{\mathrm{i}\left(\theta_{j}-t\right)}\right)=-\sum_{k \geqslant 1} \frac{\mathrm{e}^{\mathrm{i} k\left(\theta_{j}-t\right)}}{k} .
$$

Hence, for $t$ such that $Z_{X}(t) \neq 0$,

$$
\log Z_{X}(t)=-\sum_{j=1}^{N} \sum_{k \geqslant 1} \frac{\mathrm{e}^{\mathrm{i} k\left(\theta_{j}-t\right)}}{k}=-\sum_{k \geqslant 1} \frac{\mathrm{e}^{-\mathrm{i} k t}}{k} \operatorname{tr}\left(X^{k}\right)
$$


12388 Y. Barhoumi-Andréani et al.

Therefore,

$$
\operatorname{Re} \log Z_{X}(t)=-\frac{1}{2}\left(\sum_{k \geqslant 1} \frac{1}{k} \mathrm{e}^{-\mathrm{i} k t} \operatorname{tr}\left(X^{k}\right)+\sum_{k \geqslant 1} \frac{1}{k} \mathrm{e}^{\mathrm{i} k t} \operatorname{tr}\left(X^{-k}\right)\right)=-\frac{1}{2} \sum_{k \in \mathbb{Z}^{*}} \frac{1}{|k|} \mathrm{e}^{-\mathrm{i} k t} \operatorname{tr}\left(X^{k}\right)
$$

and

$$
\operatorname{Im} \log Z_{X}(t)=-\frac{1}{2 \mathrm{i}}\left(\sum_{k \geqslant 1} \frac{1}{k} \mathrm{e}^{-\mathrm{i} k t} \operatorname{tr}\left(X^{k}\right)-\sum_{k \geqslant 1} \frac{1}{k} \mathrm{e}^{\mathrm{i} k t} \operatorname{tr}\left(X^{-k}\right)\right)=-\frac{1}{2 \mathrm{i}} \sum_{k \in \mathbb{Z}^{*}} \frac{1}{k} \mathrm{e}^{-\mathrm{i} k t} \operatorname{tr}\left(X^{k}\right) .
$$

Here, the series in $k \in \mathbb{Z}^{*}=\mathbb{Z} \backslash\{0\}$ are conditionally convergent. More precisely, for $K \geqslant 1$ set

$$
S_{t}^{(K)}:=-\frac{1}{2} \sum_{k \in \mathbb{Z}^{*},|k| \leqslant K} \frac{1}{|k|} \mathrm{e}^{-\mathrm{i} k t} \operatorname{tr}\left(X^{k}\right)
$$

and

$$
S_{t}:=\operatorname{Re} \log Z_{X}(t)
$$

then $S_{t}^{(K)}$ tends almost surely to $S_{t}$ as $K$ goes to infinity.

Moreover, one has the following classical result, [8]: For all $p, q \in \mathbb{Z}$,

$$
\mathbb{E}_{U(N)}\left(\operatorname{tr}\left(X^{p}\right) \overline{\operatorname{tr}\left(X^{q}\right)}\right)=\mathbb{1}_{\{p=q\}}|p| \wedge N
$$

Hence, for $K, L \geqslant 1, t, u \in \mathbb{R}$,

$$
\begin{aligned}
\mathbb{E}_{U(N)}\left(S_{t}^{(K)} S_{u}^{(L)}\right) & =\mathbb{E}_{U(N)}\left(\frac{1}{4} \sum_{p, q \in \mathbb{Z}^{*},|p| \leqslant K,|q| \leqslant L} \frac{\mathrm{e}^{-\mathrm{i}(p t+q u)}}{|p q|} \operatorname{tr}\left(X^{p}\right) \operatorname{tr}\left(X^{q}\right)\right) \\
& =\frac{1}{4} \sum_{p, q \in \mathbb{Z}^{*},|p| \leqslant K,|q| \leqslant L} \frac{\mathrm{e}^{-\mathrm{i}(p t+q u)}}{|p q|} \mathbb{E}_{U(N)}\left(\operatorname{tr}\left(X^{p}\right) \operatorname{tr}\left(X^{q}\right)\right) \\
& =\frac{1}{4} \sum_{p, q \in \mathbb{Z}^{*},|p| \leqslant K,|q| \leqslant L} \frac{\mathrm{e}^{-\mathrm{i}(p t+q u)}}{|p q|} \mathbb{1}_{\{p=-q\}}|q| \wedge N \quad \text { (from (11)) } \\
& =\frac{1}{4} \sum_{k \in \mathbb{Z}^{*},|k| \leqslant K \wedge L} \frac{\mathrm{e}^{\mathrm{i} k(u-t)}}{k^{2}}|k| \wedge N \\
& =\frac{1}{2} \sum_{1 \leqslant k \leqslant K \wedge L} \frac{k \wedge N}{k^{2}}\left(\frac{\mathrm{e}^{\mathrm{i} k(u-t)}+\mathrm{e}^{-\mathrm{i} k(u-t)}}{2}\right) \\
& =\frac{1}{2} \sum_{1 \leqslant k \leqslant K \wedge L} \frac{k \wedge N}{k^{2}} \cos (k(u-t)) .
\end{aligned}
$$


One deduces that

$$
\begin{aligned}
\mathbb{E}_{U(N)}\left(\left(S_{t}^{(K)}-S_{t}^{(L)}\right)^{2}\right) & =\mathbb{E}_{U(N)}\left(\left(S_{t}^{(K)}\right)^{2}\right)+\mathbb{E}_{U(N)}\left(\left(S_{t}^{(L)}\right)^{2}\right)-2 \mathbb{E}_{U(N)}\left(S_{t}^{(K)} S_{t}^{(L)}\right) \\
& =\frac{1}{2} \sum_{k \geqslant 1} \frac{k \wedge N}{k^{2}} \cos (k(u-t))\left(\mathbb{1}_{\{k \leqslant K\}}+\mathbb{1}_{\{k \leqslant L\}}-2 \mathbb{1}_{\{k \leqslant K \wedge L\}}\right) \\
& =\frac{1}{2} \sum_{k \geqslant 1} \frac{k \wedge N}{k^{2}} \cos (k(u-t)) \mathbb{1}_{\{K \wedge L<k \leqslant K \vee L\}} \\
& \leqslant \frac{1}{2} \sum_{k \geqslant K \wedge L} \frac{k \wedge N}{k^{2}},
\end{aligned}
$$

which tends to zero when $K \wedge L$ goes to infinity. Hence, $S_{t}^{(K)}$ converges in $L^{2}$ when $K$ goes to infinity, and the limit is necessarily $S_{t}$. Therefore,

$$
\mathbb{E}_{U(N)}\left(S_{t} S_{u}\right)=\lim _{K \rightarrow \infty} \frac{1}{2} \sum_{1 \leqslant k \leqslant K \wedge L} \frac{k \wedge N}{k^{2}} \cos (k(u-t))=\frac{1}{2} \sum_{k \geqslant 1} \frac{k \wedge N}{k^{2}} \cos (k(u-t))
$$

The same computation with $\tilde{S}_{t}:=\operatorname{Im} \log Z_{X}(t)$ gives exactly the same equality, namely

$$
\mathbb{E}_{U(N)}\left(\tilde{S}_{t} \tilde{S}_{u}\right)=\frac{1}{2} \sum_{k \geqslant 1} \frac{k \wedge N}{k^{2}} \cos (k(u-t))
$$

It is therefore sufficient to only show the calculations for $S_{t}$, as those for $\tilde{S}_{t}$ are identical.

Setting $\alpha=\frac{\mu}{N}$ and using this last formula, we can write

$$
\begin{aligned}
\mathbb{E}_{U(N)}\left(R_{0}^{2}\right) & =\mathbb{E}_{U(N)}\left(\left(S_{\alpha}-S_{0}\right)^{2}\right)=2 \mathbb{E}_{U}\left(S_{0}^{2}-S_{\alpha} S_{0}\right) \\
& =\sum_{k \geqslant 1} \frac{k \wedge N}{k^{2}}(1-\cos (k \alpha)) \\
& =\sum_{k \geqslant 1} \frac{k \wedge N}{k^{2}}-\sum_{k \geqslant 1} \frac{k \wedge N}{k^{2}} \cos \left(\frac{k \mu}{N}\right) .
\end{aligned}
$$

We have

$$
\sum_{k \geqslant 1} \frac{k \wedge N}{k^{2}}=\sum_{k=1}^{N} \frac{1}{k}+N \sum_{k>N} \frac{1}{k^{2}}=\log N+\gamma+O\left(\frac{1}{N}\right)+N\left(\frac{1}{N}+O\left(\frac{1}{N^{2}}\right)\right)
$$


and using Euler-Maclaurin summation, we have the following result (see [9, p. 37] for the details), that uniformly on $\alpha \in[-\pi, \pi]$

$$
\begin{aligned}
\sum_{k \geqslant 1} \frac{k \wedge N}{k^{2}} \cos (k \alpha)= & -\log \left|2 \sin \left(\frac{\alpha}{2}\right)\right|+\operatorname{Ci}(N|\alpha|)+\cos (N \alpha) \\
& -\frac{\pi}{2} N|\alpha|+N \alpha \operatorname{Si}(N \alpha)+O\left(\frac{1}{N}\right),
\end{aligned}
$$

where

$$
\operatorname{Si}(z):=\int_{0}^{z} \frac{\sin x}{x} \mathrm{~d} x=\frac{\pi}{2}-\frac{\cos z}{z}+\int_{z}^{\infty} \frac{\cos x}{x^{2}} \mathrm{~d} x
$$

and

$$
\operatorname{Ci}(z)=-\int_{z}^{\infty} \frac{\cos x}{x} d x=\gamma-\log z+\int_{0}^{z} \frac{\cos x-1}{x} \mathrm{~d} x .
$$

Let us denote $f(\mu):=\log \mu+\frac{\pi}{2} \mu-\cos \mu-\operatorname{Ci}(\mu)-\mu \operatorname{Si}(\mu)$. We therefore have, for $N$ going to infinity,

$$
\begin{aligned}
\mathbb{E}_{U(N)}\left(R_{0}^{2}\right) & =\log N+1+\gamma+f(\mu)-\log \mu+\log \left|2 \sin \left(\frac{\mu}{2 N}\right)\right|+O\left(\frac{1}{N}\right) \\
& =\log N+1+\gamma+f(\mu)-\log \mu+\log \left(2 \frac{\mu}{2 N}\left(1+O\left(\left(\frac{\mu}{N}\right)^{2}\right)\right)\right)+O\left(\frac{1}{N}\right) \\
& =1+\gamma+f(\mu)+O\left(\left(\frac{\mu}{N}\right)^{2}\right)+O\left(\frac{1}{N}\right) .
\end{aligned}
$$

Let us now study the behavior of the function $f$.

$$
\begin{aligned}
f(\mu) & =\log \mu-\cos \mu+\mu\left(\frac{\pi}{2}-\operatorname{Si}(\mu)\right)-\operatorname{Ci}(\mu) \\
& =\log \mu-\cos \mu+\mu\left(\frac{\cos \mu}{\mu}-\int_{\mu}^{\infty} \frac{\cos x}{x^{2}} \mathrm{~d} x\right)-\left(\gamma-\log \mu+\int_{0}^{\mu} \frac{\cos x-1}{x} \mathrm{~d} x\right) \\
& =-\gamma-\mu \int_{\mu}^{\infty} \frac{\cos x}{x^{2}} \mathrm{~d} x+\int_{0}^{\mu} \frac{\cos x-1}{x} \mathrm{~d} x .
\end{aligned}
$$

Bounding the integrals uniformly in $\mu>0$, one has that

$$
\begin{aligned}
f(\mu) & =-\gamma-\mu O\left(\int_{\mu}^{\infty} \frac{1}{x^{2}} \mathrm{~d} x\right)+O\left(\int_{0}^{\mu}\left(\frac{1}{x} \wedge 1\right) \mathrm{d} x\right) \\
& =-\gamma+O(1)+O(1+\log (\mu \vee 1))=O\left(\log \left(\frac{\mu}{2 \pi} \vee 2\right)\right),
\end{aligned}
$$

where we have used the standard notation $\mu \vee 1$ to mean the maximum of $\mu$ and 1 . 
Substituting this into (12) yields

$$
\mathbb{E}_{U(N)}\left(R_{0}^{2}\right)=O\left(\log \left(\frac{\mu}{2 \pi} \vee 2\right)\right)=O(\log M)
$$

\subsection{Control in probability of the mean oscillation of the logarithm of the characteristic polynomials}

Lemma 3.8. Given $n \in \mathbb{N}$, consider an i.i.d. sequence $\left(U_{j}\right)_{1 \leqslant j \leqslant n}$ of random matrices following the Haar measure on $\mathrm{SU}(N)$, and let

$$
L_{j}(\theta):=\frac{\log \left|Z_{U_{j}}(\theta)\right|}{\sqrt{\frac{1}{2} \log N}} .
$$

For $\delta \in\left(0, \frac{1}{2}\right)$, consider the random set

$$
\mathscr{E}_{\delta}:=\bigcup_{i=1}^{n}\left(\left\{\theta \in[0,2 \pi] /\left|L_{i}(\theta)\right| \geqslant \delta^{-1}\right\} \cup \bigcup_{j \neq i}\left\{\theta \in[0,2 \pi] /\left|L_{j}(\theta)-L_{i}(\theta)\right| \leqslant \delta\right\}\right)
$$

There exists $C_{3}>0$, depending only on $n$, such that for all $N \geqslant 4$

$$
\mathbb{E}\left(\lambda_{2 \pi}\left(\mathscr{E}_{\delta}\right)\right) \leqslant c_{3} \delta \log (1 / \delta)
$$

where $\lambda_{2 \pi}$ denotes the normalized Lebesgue measure on $[0,2 \pi]$.

Proof. One has

$$
\begin{aligned}
\mathbb{E}_{\mathrm{SU}(N)}\left(\lambda_{2 \pi}\left(\mathscr{E}_{\delta}\right)\right) & =\int_{0}^{2 \pi} \frac{\mathrm{d} \theta}{2 \pi} \mathbb{P}_{\mathrm{SU}(N)}\left(\theta \in \mathscr{E}_{\delta}\right) \\
& \leqslant \sum_{i=1}^{n} \int_{0}^{2 \pi} \frac{\mathrm{d} \theta}{2 \pi} \mathbb{P}_{\mathrm{SU}(N)}\left(\left|L_{i}(\theta)\right| \geqslant \delta^{-1}\right)+\sum_{1 \leqslant i \neq j \leqslant n} \int_{0}^{2 \pi} \frac{\mathrm{d} \theta}{2 \pi} \mathbb{P}_{\mathrm{SU}(N)}\left(\left|L_{j}(\theta)-L_{i}(\theta)\right| \leqslant \delta\right) .
\end{aligned}
$$

In the last second sum, since $i \neq j, L_{i}(\theta)$ is independent of $L_{j}(\theta)$, and hence, conditioning on $L_{i}(\theta)$, one gets

$$
\mathbb{P}_{\mathrm{SU}(N)}\left(\left|L_{j}(\theta)-L_{i}(\theta)\right| \leqslant \delta\right)=\mathbb{E}_{\mathrm{SU}(N)}\left(H\left(L_{i}(\theta)\right)\right),
$$

where

$$
H(X)=\mathbb{P}_{\mathrm{SU}(N)}\left(\left|L_{j}(\theta)-X\right| \leqslant \delta\right) .
$$


One deduces that for all $i \neq j$

$$
\mathbb{P}_{\mathrm{SU}(N)}\left(\left|L_{j}(\theta)-L_{i}(\theta)\right| \leqslant \delta\right) \leqslant \sup _{\theta \in[0,2 \pi], x \in \mathbb{R}} \mathbb{P}_{\mathrm{SU}(N)}\left(|\log | Z_{X}(\theta)|-x| \leqslant \delta \sqrt{\frac{1}{2} \log N}\right) .
$$

Using Markov's inequality, that is, $\mathbb{P}(X \geqslant t) \leqslant \mathbb{E}(X) / t$ for all $t>0$ and all random variables $X \geqslant 0$, one thus obtains

$$
\begin{aligned}
\mathbb{E}_{\mathrm{SU}(N)}\left(\lambda_{2 \pi}\left(\mathscr{E}_{\delta}\right)\right) \leqslant & n \int_{0}^{2 \pi} \frac{\mathrm{d} \theta}{2 \pi} \mathbb{P}_{\mathrm{SU}(N)}\left(|\log | Z_{X}(\theta)|| \geqslant \delta^{-1} \sqrt{\frac{1}{2} \log N}\right) \\
& +n(n-1) \sup _{\theta \in[0,2 \pi], x \in \mathbb{R}} \mathbb{P}_{\mathrm{SU}(N)}\left(|\log | Z_{X}(\theta)|-x| \leqslant \delta \sqrt{\frac{1}{2} \log N}\right) \\
\leqslant & n c_{1} \mathrm{e}^{-\frac{\delta^{-1}}{\sqrt{2}}\left(\frac{\delta^{-1}}{\sqrt{2}} \wedge \frac{\sqrt{\log N}}{2}\right)}+n(n-1) C(\delta / \sqrt{2}) \log (\sqrt{2} / \delta) .
\end{aligned}
$$

Now,

$$
\mathrm{e}^{-\frac{\delta^{-1}}{\sqrt{2}}\left(\frac{\delta^{-1}}{\sqrt{2}} \wedge \frac{\sqrt{\log N}}{2}\right)} \leqslant \mathrm{e}^{-\frac{\delta^{-1}}{\sqrt{2}}\left(\frac{2}{\sqrt{2}} \wedge \frac{\sqrt{\log 2}}{2}\right)} \leqslant \mathrm{e}^{-\frac{\delta^{-1}}{5}}=O(\delta \log (1 / \delta))
$$

and

$$
\delta \log (\sqrt{2} / \delta) \leqslant \delta \log \left(\sqrt{\delta^{-1}} / \delta\right)=\frac{3 \delta}{2} \log (1 / \delta),
$$

which gives Lemma 3.8.

\subsection{Control in expectation of the oscillation of the logarithm of the characteristic polynomials on a small period}

In what follows, assume the dimension $N \geqslant 4$. Let $K$ be an integer such that $2 \leqslant K \leqslant N / 2$, defined as a function of $N$ which is asymptotically equivalent to $N /(\log N)^{3 / 64}$ when $N$ goes to infinity. We denote

$$
M:=N / K \geqslant 2
$$

which is asymptotic to $(\log N)^{3 / 64}$, and we also define a parameter $\delta \in\left(0, \frac{1}{4}\right)$ as a function of $N$, asymptotic to $(\log N)^{-3 / 32}$ when $N$ goes to infinity. For $\theta_{0} \in[0,2 \pi]$, we denote, for $0 \leqslant k \leqslant K$.

$$
\theta_{k}:=\theta_{0}+\frac{2 \pi k}{K}=\theta_{0}+\frac{2 \pi k M}{N},
$$

and for $0 \leqslant k \leqslant K-1$,

$$
\Delta:=\theta_{k+1}-\theta_{k}=\frac{2 \pi}{K}=\frac{2 \pi M}{N} .
$$


The angle $\theta_{0}$ is chosen in such a way that the following technical condition is satisfied:

$$
\begin{aligned}
& \sum_{k=0}^{K-1} \mathbb{E}_{\mathrm{SU}(N)}\left(\left|\operatorname{Im} \log Z_{X}\left(\theta_{k}+(1-\sqrt{\delta}) \Delta\right)-\operatorname{Im} \log Z_{X}\left(\theta_{k}+\sqrt{\delta} \Delta\right)\right|\right) \\
& \quad \leqslant K \mathbb{E}_{\mathrm{SU}(N)}\left(\int_{0}^{2 \pi} \frac{\mathrm{d} \theta}{2 \pi}\left|\operatorname{Im} \log Z_{X}(\theta+(1-\sqrt{\delta}) \Delta)-\operatorname{Im} \log Z_{X}(\theta+\sqrt{\delta} \Delta)\right|\right) .
\end{aligned}
$$

This choice is always possible. Indeed, if the converse (strict) inequality were true for all $\theta_{0}$, then one would get a contradiction by integrating with respect to $\theta_{0} \in[0,2 \pi / K)$. We then define the interval $J:=\left[\theta_{0}, \theta_{0}+2 \pi\right)=\left[\theta_{0}, \theta_{K}\right)$. Note that all the objects introduced here can be defined only as a function of $N$. Moreover, by applying Lemma 3.7 to $\theta+\sqrt{\delta} \Delta$ and $\mu=N(1-2 \sqrt{\delta}) \Delta \leqslant 2 \pi M$, we deduce that the assumption made on $\theta_{0}$ implies that

$$
\sum_{k=0}^{K-1} \mathbb{E}_{\mathrm{SU}(N)}\left(\left|\operatorname{Im} \log Z_{X}\left(\theta_{k}+(1-\sqrt{\delta}) \Delta\right)-\operatorname{Im} \log Z_{X}\left(\theta_{k}+\sqrt{\delta} \Delta\right)\right|\right)=O(K \sqrt{\log M}) .
$$

We now introduce the 2-oscillation of the real and imaginary parts of the logarithm of the characteristic polynomial.

Definition 3.9. For $\theta \in J$ and $\mu \in[0,2 \pi M]$, and for the canonical matrix $X \in U(N)$, the 2-oscillations of $\operatorname{Re} \log Z_{X}$ and $\operatorname{Im} \log Z_{X}$ are defined by

$$
\begin{aligned}
\Delta_{\mu} R_{\theta} & :=\frac{1}{\sqrt{\log (M)}}\left|\operatorname{Re} \log Z_{X}\left(\theta+\frac{\mu}{N}\right)-\operatorname{Re} \log Z_{X}(\theta)\right|, \\
\Delta_{\mu} I_{\theta} & :=\frac{1}{\sqrt{\log (M)}}\left|\operatorname{Im} \log Z_{X}\left(\theta+\frac{\mu}{N}\right)-\operatorname{Im} \log Z_{X}(\theta)\right| .
\end{aligned}
$$

In case of several matrices $\left(X_{j}\right)_{1 \leqslant j \leqslant n}$, we denote the corresponding 2-oscillations by $\Delta_{\mu} R_{\theta}^{(j)}$ and $\Delta_{\mu} I_{\theta}^{(j)}$.

Now, we need to introduce several random sets. The most important ones can be informally described as follows:

(1) A set $\mathscr{N}_{1}$ of indices $k$ such that the average of the 2-oscillations $\Delta_{\mu} R_{\theta}$ and $\Delta_{\mu} I_{\theta}$ of the logarithm of the characteristic polynomials for $\theta \in\left[\theta_{k}, \theta_{k+1}\right]$ and $\mu \in[0,2 \pi M]$ is sufficiently small.

(2) For $k \in \mathscr{N}_{1}$, a subset $\mathscr{G}_{k}$ of $\left[\theta_{k}, \theta_{k+1}\right]$ for which the average of the 2-oscillations with respect to $\mu \in[0,2 \pi M]$ is small enough.

(3) A subset $\mathscr{N}_{2}$ of $\mathscr{N}_{1}$ of "good" indices, such that there exists $\theta \in\left[\theta_{k}, \theta_{k}+\sqrt{\delta} \Delta\right]$, both in $\mathscr{G}_{k}$ and $\mathscr{E}_{\delta} c$. This last set, introduced in Lemma 3.8, corresponds to the 
fact that the logarithms of the absolute values of the characterize polynomials are not too large and not too close from each other. It is from this last condition that we can define the "carrier wave".

(4) For $k \in \mathscr{N}_{2}$, and for some $\theta_{k}^{*} \in\left[\theta_{k}, \theta_{k}+\sqrt{\delta} \Delta\right] \cap \mathscr{G}_{k} \cap \mathscr{E}_{\delta}$, a subset $\mathscr{Y}_{k}$ of $[0,2 \pi M]$ such that the 2-oscillations $\Delta_{\mu} R_{\theta_{k}^{*}}$ and $\Delta_{\mu} I_{\theta_{k}^{*}}$ are sufficiently small. This condition will ensure that the carrier wave index corresponding to $\theta=\theta_{k}^{*}+\mu / N$ does not depend on $\mu \in \mathscr{Y}_{k}$.

(5) From this property, we deduce that, for each pair of consecutive gaps between zeros of the carrier wave, which are sufficiently large to contain an angle of the form $\theta_{k}^{*}+\mu / N$ for $k \in \mathscr{N}_{2}$ and $\mu \in \mathscr{Y}_{k}$ ("roomy gaps"), one can find, with the notation of the introduction, a sign change of $\mathrm{i}^{N} \mathrm{e}^{\mathrm{i} N \theta / 2} F_{N}\left(\mathrm{e}^{-\mathrm{i} \theta}\right)$, and hence a zero of $F_{N}$.

All these sets will be precisely defined later in this paper. They are constructed in a way such that their measure is "large" with "high" probability (again, in a manner to be made precise). The corresponding estimates will then be used to prove our main result, Theorem 1.1.

Lemma 3.10. Let $\mathbb{P}_{\mathrm{SU}(N)}^{(n)}$ be the $n$-fold product of the Haar measure on $\mathrm{SU}(N), \mathbb{E}_{\mathrm{SU}(N)}^{(n)}$ the corresponding expectation, and $\left(X_{j}\right)_{1 \leqslant j \leqslant n}$ be the canonical sequence of $n$ matrices in $\mathrm{SU}(N)$. Then:

(1) There exists a random set $\mathscr{N}_{1} \subset \llbracket 0, K-1 \rrbracket$ such that $\mathbb{E}_{\mathrm{SU}(N)}^{(n)}\left(\left|\mathscr{N}_{1}\right|\right) \geqslant(1-\delta) K$ and $\mathbb{P}_{\mathrm{SU}(N)}^{(n)}$-a.s., $\forall(j, k) \in \llbracket 1, n \rrbracket \times \mathscr{N}_{1}$,

$$
\int_{\theta_{k}}^{\theta_{k+1}} \int_{0}^{2 \pi M} \Delta_{\mu} R_{\theta}^{(j)} \frac{\mathrm{d} \mu}{2 \pi M} \frac{\mathrm{d} \theta}{2 \pi}=O\left(\frac{1}{\delta K}\right)
$$

and

$$
\int_{\theta_{k}}^{\theta_{k+1}} \int_{0}^{2 \pi M} \Delta_{\mu} I_{\theta}^{(j)} \frac{\mathrm{d} \mu}{2 \pi M} \frac{\mathrm{d} \theta}{2 \pi}=O\left(\frac{1}{\delta K}\right) .
$$

(2) $\mathbb{P}_{\mathrm{SU}(N)}^{(n)}$-a.s., $\forall k \in \mathscr{N}_{1}, \exists \mathscr{G}_{k} \subset\left[\theta_{k}, \theta_{k+1}\right)$ such that $\lambda_{2 \pi}\left(\mathscr{G}_{k}\right) \geqslant(1-\delta) / K$ and, $\forall \theta \in$ $\mathscr{G}_{k}, j \in \llbracket 1, n \rrbracket$,

$$
\int_{0}^{2 \pi M} \Delta_{\mu} R_{\theta}^{(j)} \frac{\mathrm{d} \mu}{2 \pi M}=O\left(\frac{1}{\delta^{2}}\right) \text { and } \int_{0}^{2 \pi M} \Delta_{\mu} I_{\theta}^{(j)} \frac{\mathrm{d} \mu}{2 \pi M}=O\left(\frac{1}{\delta^{2}}\right) .
$$

Here, the implied constant in the $O(\cdot)$ symbols depends only on $n$. 
Proof. By (13) and the similar estimate for the imaginary part, we have uniformly (with a universal implied constant),

$$
\mathbb{E}_{U(N)}\left(\left(\Delta_{\mu} R_{0}\right)^{2}\right)+\mathbb{E}_{U(N)}\left(\left(\Delta_{\mu} I_{0}\right)^{2}\right)=O(1)
$$

The Cauchy-Schwarz inequality ensures that

$$
\mathbb{E}_{U(N)}\left(\Delta_{\mu} R_{0}\right)+\mathbb{E}_{U(N)}\left(\Delta_{\mu} I_{0}\right)=O(1)
$$

that is,

$$
\int_{J} \mathbb{E}_{\mathrm{SU}(N)}\left(\Delta_{\mu} R_{\theta}+\Delta_{\mu} I_{\theta}\right) \frac{\mathrm{d} \theta}{2 \pi}=O(1)
$$

which implies

$$
\int_{J} \int_{0}^{2 \pi M} \mathbb{E}_{\mathrm{SU}(N)}\left(\Delta_{\mu} R_{\theta}+\Delta_{\mu} I_{\theta}\right) \frac{\mathrm{d} \mu}{2 \pi M} \frac{\mathrm{d} \theta}{2 \pi}=O(1) .
$$

Splitting the interval $J$ into $K$ equal pieces and applying this estimate to $n$ independent matrices $\left(X_{j}\right)_{1 \leqslant j \leqslant n}$ following the Haar measure on $\mathrm{SU}(N)$, one gets

$$
\frac{1}{n} \sum_{j=1}^{n} \sum_{k=0}^{K-1} \mathbb{E}_{\mathrm{SU}(N)}^{(n)}\left(\int_{\theta_{k}}^{\theta_{k+1}} \int_{0}^{2 \pi M}\left(\Delta_{\mu} R_{\theta}^{(j)}+\Delta_{\mu} I_{\theta}^{(j)}\right) \frac{\mathrm{d} \mu}{2 \pi M} \frac{\mathrm{d} \theta}{2 \pi}\right)=O(1) .
$$

Applying Markov's inequality, we deduce that there exists a universal constant $\kappa>0$, such that

$$
\mathbb{E}_{\mathrm{SU}(N)}^{(n)}\left(\operatorname{card}\left\{(j, k) \in \llbracket 1, n \rrbracket \times \llbracket 0, K-1 \rrbracket: \int_{\theta_{k}}^{\theta_{k+1}} \int_{0}^{2 \pi M} \Delta_{\mu} R_{\theta}^{(j)} \frac{\mathrm{d} \mu}{2 \pi M} \frac{\mathrm{d} \theta}{2 \pi} \geqslant \frac{\kappa n}{K \delta}\right\}\right) \leqslant \frac{\delta K}{2}
$$

and

$$
\mathbb{E}_{\mathrm{SU}(N)}^{(n)}\left(\operatorname{card}\left\{(j, k) \in \llbracket 1, n \rrbracket \times \llbracket 0, K-1 \rrbracket: \int_{\theta_{k}}^{\theta_{k+1}} \int_{0}^{2 \pi M} \Delta_{\mu} I_{\theta}^{(j)} \frac{\mathrm{d} \mu}{2 \pi M} \frac{\mathrm{d} \theta}{2 \pi} \geqslant \frac{\kappa n}{K \delta}\right\}\right) \leqslant \frac{\delta K}{2} .
$$

Setting

$$
\mathscr{N}_{1}:=\bigcap_{j=1}^{n}\left\{k \in \llbracket 0, K-1 \rrbracket: \int_{\theta_{k}}^{\theta_{k+1}} \int_{0}^{2 \pi M} \Delta_{\mu} R_{\theta}^{(j)} \frac{\mathrm{d} \mu}{2 \pi M} \frac{\mathrm{d} \theta}{2 \pi} \leqslant \frac{\kappa n}{K \delta}, \int_{\theta_{k}}^{\theta_{k+1}} \int_{0}^{2 \pi M} \Delta_{\mu} I_{\theta}^{(j)} \frac{\mathrm{d} \mu}{2 \pi M} \frac{\mathrm{d} \theta}{2 \pi} \leqslant \frac{\kappa n}{K \delta}\right\}
$$

we thus get

$$
\mathbb{E}_{\mathrm{SU}(N)}^{(n)}\left(\left|\mathscr{N}_{1}\right|\right) \geqslant(1-\delta) K .
$$

Now, for $k \in \mathscr{N}_{1}$, let us set

$$
\mathscr{G}_{k}:=\left[\theta_{k}, \theta_{k+1}\right) \cap \bigcap_{j=1}^{n}\left\{\int_{0}^{2 \pi M} \Delta_{\mu} R^{(j)} \frac{\mathrm{d} \mu}{2 \pi M} \leqslant \frac{2 \kappa n^{2}}{\delta^{2}}, \int_{0}^{2 \pi M} \Delta_{\mu} I^{(j)} \frac{\mathrm{d} \mu}{2 \pi M} \leqslant \frac{2 \kappa n^{2}}{\delta^{2}}\right\} .
$$


12396 Y. Barhoumi-Andréani et al.

Applying Markov's inequality again, we get that $\mathbb{P}_{\mathrm{SU}(N)}^{(n)}$-a.s. we have

$$
\lambda_{2 \pi}\left(\mathscr{G}_{k}\right) \geqslant(1-\delta) / K
$$

Definition 3.11 (Good indices). An index $k \in \llbracket 0, K-1 \rrbracket$ is said to be good if:

(1) $k \in \mathscr{N}_{1}$,

(2) $\mathscr{E}_{\delta}^{C} \cap \mathscr{G}_{k} \cap\left[\theta_{k}, \theta_{k}+\sqrt{\delta} \Delta\right) \neq \emptyset$.

We denote by $\mathscr{N}_{2}$ the set of good indices, that is,

$$
\mathscr{N}_{2}:=\left\{k \in \mathscr{N}_{1} / \mathscr{E}_{\delta}^{C} \cap \mathscr{G}_{k} \cap\left[\theta_{k}, \theta_{k}+\sqrt{\delta} \Delta\right) \neq \emptyset\right\} .
$$

An index is said to be bad if it is not good.

Lemma 3.12. With the notation above, the set of good indices satisfies

$$
\mathbb{E}_{\mathrm{SU}(N)}^{(n)}\left(\left|\mathscr{N}_{2}\right|\right)=K(1-O(\sqrt{\delta} \log (1 / \delta))),
$$

where the implied constant in the $O(\cdot)$ symbol depends only on $n$.

Proof. If $k \in \mathscr{N}_{2}^{c}$, either $k \in \mathscr{N}_{1}^{c}$, or $k \in \mathscr{N}_{1}$ and $\mathscr{E}_{\delta}^{c} \cap \mathscr{G}_{k} \cap\left[\theta_{k}, \theta_{k}+\sqrt{\delta} \Delta\right)=\emptyset$. This is, $\mathscr{N}_{2}^{c}=\mathscr{N}_{1}^{c} \cup \tilde{\mathscr{N}}_{1}$ where

$$
\tilde{\mathscr{N}}_{1}:=\left\{k \in \mathscr{N}_{1}: \mathscr{G}_{k} \cap\left[\theta_{k}, \theta_{k}+\sqrt{\delta} \Delta\right) \subset \mathscr{E}_{\delta}\right\}
$$

By (17), we have $\mathbb{E}_{\mathrm{SU}(N)}^{(n)}\left(\left|\mathscr{N}_{1}^{C}\right|\right) \leqslant \delta K$.

For all $k \in \tilde{\mathscr{N}}_{1}$, we have $\mathscr{E}_{\delta} \supset \mathscr{G}_{k} \cap\left[\theta_{k}, \theta_{k}+\sqrt{\delta} \Delta\right)$, that is, $\mathscr{E}_{\delta} \supset \bigcup_{k \in \tilde{\mathscr{N}}_{1}} \mathscr{G}_{k} \cap$ $\left[\theta_{k}, \theta_{k}+\sqrt{\delta} \Delta\right)$, where the union is disjoint, and thus, $\lambda_{2 \pi}\left(\mathscr{E}_{\delta}\right) \geqslant\left|\tilde{\mathscr{N}}_{1}\right| \min _{k} \lambda_{2 \pi}\left(\mathscr{G}_{k} \cap\left[\theta_{k}\right.\right.$, $\left.\left.\theta_{k}+\sqrt{\delta} \Delta\right)\right)$.

By (18), we have, $\mathbb{P}_{\mathrm{SU}(N)}^{(n)}$-a.s.,

$$
\begin{aligned}
\lambda_{2 \pi}\left(\mathscr{G}_{k} \cap\left[\theta_{k}, \theta_{k}+\sqrt{\delta} \Delta\right)\right) & \geqslant \lambda_{2 \pi}\left(\mathscr{G}_{k}\right)+\lambda_{2 \pi}\left(\left[\theta_{k}, \theta_{k}+\sqrt{\delta} \Delta\right)\right)-\lambda_{2 \pi}\left(\left[\theta_{k}, \theta_{k}+\Delta\right)\right) \\
& \geqslant \frac{1}{K}((1-\delta)+\sqrt{\delta}-1) .
\end{aligned}
$$

Now, since $\delta<\frac{1}{4}$, we obtain $\mathbb{P}_{\mathrm{SU}(N)}^{(n)}$-a.s.

$$
\lambda_{2 \pi}\left(\mathscr{G}_{k} \cap\left[\theta_{k}, \theta_{k}+\sqrt{\delta} \Delta\right)\right) \geqslant \frac{\sqrt{\delta}}{2 K} .
$$


This implies that $\mathbb{P}_{\mathrm{SU}(N)}^{(n)}$-a.s.

$$
\left|\tilde{\mathscr{N}}_{1}\right| \leqslant \frac{2 K}{\sqrt{\delta}} \lambda_{2 \pi}\left(\mathscr{E}_{\delta}\right)
$$

Now, by Lemma (3.8), $\mathbb{E}_{\mathrm{SU}(N)}^{(n)}\left(\left|\tilde{\mathscr{N}}_{1}\right|\right)=O(K \sqrt{\delta} \log (1 / \delta))$ and so

$$
\mathbb{E}_{\mathrm{SU}(N)}^{(n)}\left(\left|\mathscr{N}_{2}^{c}\right|\right) \leqslant \mathbb{E}_{\mathrm{SU}(N)}^{(n)}\left(\left|\mathscr{N}_{1}^{c}\right|\right)+\mathbb{E}_{\mathrm{SU}(N)}^{(n)}\left(\left|\tilde{\mathscr{N}}_{1}\right|\right) \leqslant \delta K+O(K \sqrt{\delta} \log (1 / \delta))
$$

\subsection{Speed of the good oscillation of the logarithm of the characteristic polynomials}

Lemma 3.13. With the notation above, and $\mathbb{P}_{\mathrm{SU}(N)}^{(n)}$-a.s., $\forall k \in \mathscr{N}_{2}$, there exists a random set $\mathscr{Y}_{k} \subset[0,2 \pi M]$, and $\theta_{k}^{*} \in \mathscr{E}_{\delta}^{c} \cap \mathscr{G}_{k} \cap\left[\theta_{k}, \theta_{k}+\sqrt{\delta} \Delta\right)$, such that

$$
\lambda_{2 \pi M}\left(\mathscr{Y}_{k}\right)=1-O\left(\delta^{-2}(\log N)^{-1 / 4}(\log M)^{1 / 2}\right)
$$

where $\lambda_{2 \pi M}$ is $1 /(2 \pi M)$ times the Lebesgue measure, and for all $j \in \llbracket 1, n \rrbracket, \mu \in \mathscr{Y}_{k}$,

$$
\Delta_{\mu} R_{\theta_{k}^{*}}^{(j)}=O\left(\frac{(\log N)^{1 / 4}}{(\log M)^{1 / 2}}\right) \quad \text { and } \quad \Delta_{\mu} I_{\theta_{k}^{*}}^{(j)}=O\left(\frac{(\log N)^{1 / 4}}{(\log M)^{1 / 2}}\right) .
$$

Again, the implied constant in the $O(\cdot)$ symbol depends only on $n$.

Proof. Let $k \in \mathscr{N}_{2}$ and $\theta_{k}^{*} \in \mathscr{E}_{\delta}^{C} \cap \mathscr{G}_{k} \cap\left[\theta_{k}, \theta_{k}+\sqrt{\delta} \Delta\right)$. Set

$$
\mathscr{Y}_{k}:=\bigcap_{j=1}^{n}\left\{\Delta . R_{\theta_{k}^{*}}^{(j)} \leqslant \varepsilon, \Delta . I_{\theta_{k}^{*}}^{(j)} \leqslant \varepsilon\right\}
$$

where

$$
\varepsilon:=\frac{(\log N)^{1 / 4}}{(\log M)^{1 / 2}}
$$

Applying Markov's inequality, we get

$$
\begin{aligned}
\lambda_{2 \pi M}\left(\mathscr{Y}_{k}^{c}\right) & \leqslant \lambda_{2 \pi M}\left(\bigcup_{j=1}^{n}\left\{\Delta . R_{\theta_{k}^{*}}^{(j)} \geqslant \varepsilon\right\}\right)+\lambda_{2 \pi} M\left(\bigcup_{j=1}^{n}\left\{\Delta_{.} I_{\theta_{k}^{*}}^{(j)} \geqslant \varepsilon\right\}\right) \\
& \leqslant \frac{2 n}{\varepsilon} \max _{1 \leqslant j \leqslant n}\left(\int_{0}^{2 \pi M} \Delta_{\mu} R_{\theta_{k}^{*}}^{(j)} \frac{\mathrm{d} \mu}{2 \pi M} \vee \int_{0}^{2 \pi M} \Delta_{\mu} I_{\theta_{k}^{*}}^{(j)} \frac{\mathrm{d} \mu}{2 \pi M}\right)=\frac{1}{\varepsilon} O\left(\delta^{-2}\right),
\end{aligned}
$$

by (15), which gives the announced result. 


\subsection{The number of sign changes}

Let us go back to Theorem 1.1. We need to estimate the number of zeros of $F_{N}$ on the unit circle, or equivalently, the number of values of $\theta \in J$ such that the following quantity vanishes:

$$
\mathrm{i}^{N} \mathrm{e}^{\mathrm{i} N \theta / 2} F_{N}\left(\mathrm{e}^{-\mathrm{i} \theta}\right)=\mathrm{i}^{N} \mathrm{e}^{\mathrm{i} N \theta / 2} \sum_{j=1}^{n} b_{j} \Phi_{U_{N, j}}\left(\mathrm{e}^{-\mathrm{i} \theta}\right)=\sum_{j=1}^{n} b_{j} \mathrm{i}^{N} \mathrm{e}^{\mathrm{i} N \theta / 2} Z_{U_{N, j}}(\theta) .
$$

Using the fact that $U_{N, j} \in \mathrm{SU}(N)$, one checks that $\mathrm{i}^{N} \mathrm{e}^{\mathrm{i} N \theta / 2} Z_{U_{N, j}}(\theta)$ is real, and then the number of zeros of $F_{N}$ on the unit circle is bounded from below by the number of sign changes, when $\theta$ increases from $\theta_{0}$ to $\theta_{0}+2 \pi$, of the real quantity given by the right-hand side of (22). Now, the order of magnitude of $\log \left|Z_{U_{N, j}}(\theta)\right|$ is $\sqrt{\log N}$ and more precisely, Lemma 3.8 informally means that for most values of $\theta$, the values of $\log \left|Z_{U_{N, j}}(\theta)\right|$ for $1 \leqslant j \leqslant n$ are pairwise separated by an interval of length of order $\sqrt{\log N}$. Hence, one of the terms in the sum at the right-hand side of (22) should dominate all the others. If $j_{0}$ is the corresponding index, one can expect that the sign changes of (22) can, at least locally, be related to the corresponding sign changes of $\mathrm{i}^{N} \mathrm{e}^{\mathrm{i} N \theta / 2} Z_{U_{N, j_{0}}}(\theta)$, which are associated to the zeros of the characteristic polynomial $Z_{U_{N, j_{0}}}$. This should give a lower bound on the number of sign changes of (22).

This informal discussion motivates the following definition.

Definition 3.14. With the notation of the previous subsections, for all $k \in \mathscr{N}_{2}$, we define the carrier wave index by:

$$
j_{k}:=\operatorname{Arg} \max _{j}\left\{\operatorname{Re} \log Z_{X_{j}}\left(\theta_{k}^{*}\right)\right\},
$$

where $\theta_{k}^{*}$ is the random angle introduced in Lemma 3.13. Moreover, to each $k \in \mathscr{N}_{2}$ we associate the interval

$$
J_{k}:=\left[\theta_{k}^{*}, \theta_{k}^{*}+(1-\sqrt{\delta}) \Delta\right]
$$

As $\theta_{k}^{*} \in \mathscr{E}_{\delta}^{C}$, we have that for all $j \neq j_{k}, \operatorname{Re} \log Z_{X_{j}}\left(\theta_{k}^{*}\right) \leqslant \operatorname{Re} \log Z_{X_{j_{k}}}\left(\theta_{k}^{*}\right)-\frac{\delta}{\sqrt{2}} \sqrt{\log N}$. From (21), we deduce that for all $j \neq j_{k}$ and for all $\mu \in \mathscr{Y}_{k}$ we have

$$
\operatorname{Re} \log Z_{X_{j}}\left(\theta_{k}^{*}+\frac{\mu}{N}\right) \leqslant \operatorname{Re} \log Z_{X_{j_{k}}}\left(\theta_{k}^{*}+\frac{\mu}{N}\right)-\frac{\delta}{\sqrt{2}}(\log N)^{1 / 2}+O\left((\log N)^{1 / 4}\right) .
$$

Now, since

$$
1 / \delta=O\left((\log N)^{1 / 10}\right)
$$


with a universal implied constant, we then get, for a universal $c>0$,

$$
\frac{\left|Z_{X_{j}}\left(\theta_{k}^{*}+\frac{\mu}{N}\right)\right|}{\left|Z_{X_{j_{k}}}\left(\theta_{k}^{*}+\frac{\mu}{N}\right)\right|} \leqslant \exp \left(-2 C(\log N)^{4 / 10}+O\left((\log N)^{1 / 4}\right)\right) \leqslant \exp \left(-C(\log N)^{4 / 10}\right),
$$

for $N$ large enough, depending only on $n$. This implies

$$
\begin{aligned}
\left|\sum_{j \neq j_{k}} b_{j} Z_{X_{j}}\left(\theta_{k}^{*}+\frac{\mu}{N}\right)\right| & \leqslant \frac{\sum_{j}\left|b_{j}\right|}{\min _{j}\left|b_{j}\right|}\left|b_{j_{k}} Z_{X_{j_{k}}}\left(\theta_{k}^{*}+\frac{\mu}{N}\right)\right| \exp \left(-c(\log N)^{4 / 10}\right) \\
& \leqslant \frac{1}{2}\left|b_{j_{k}} Z_{X_{j_{k}}}\left(\theta_{k}^{*}+\frac{\mu}{N}\right)\right|
\end{aligned}
$$

for $N \geqslant N_{0}$, where $N_{0}$ depends only on $n, b_{1}, \ldots, b_{n}$. Hence, for $k \in \mathscr{N}_{2}, \mu \in \mathscr{Y}_{k}$, and $\theta=\theta_{k}^{*}+\mu / N$, the quantity

$$
G(\theta):=\sum_{j=1}^{n} b_{j} \mathrm{i}^{N} \mathrm{e}^{\mathrm{i} N \theta / 2} Z_{X_{j}}(\theta)
$$

which is $\mathbb{P}_{\mathrm{SU}(N)}^{(n)}$-a.s. real, has the same sign as its term of index $j_{k}$.

Theorem 1.1 is proved if we show that the expectation of number of sign changes of $G(\theta)$ for $\theta \in J$, under $\mathbb{P}_{\mathrm{SU}(N)}^{(n)}$, is bounded from below by $N-O(N)$. Hence, it is sufficient to get

$$
\mathbb{E}_{\mathrm{SU}(N)}^{(n)}\left(\sum_{k \in N_{2}} \mathcal{S}_{k}\right) \geqslant N-o(N)
$$

where $\mathcal{S}_{k}$ is the number of sign changes of $b_{j_{k}} \mathrm{i}^{N} \mathrm{e}^{\mathrm{i} N \theta / 2} Z_{X_{j_{k}}}(\theta)$, for $\theta \in J_{k} \cap\left\{\theta_{k}^{*}+\frac{\mu}{N}, \mu \in \mathscr{Y}_{k}\right\}$.

Now, for $k \in \mathscr{N}_{2}$, let $\alpha_{k, 1} \leqslant \alpha_{k, 2} \leqslant \cdots \leqslant \alpha_{k, v_{k}}$ be the eigenangles, counted with multiplicity, of $X_{j_{k}}$ in the interval $J_{k}$. The sign of $b_{j} \mathrm{i}^{N} \mathrm{e}^{\mathrm{i} N \theta / 2} Z_{X_{j_{k}}}$ alternates between the different intervals $\left(\alpha_{k, 1}, \alpha_{k, 2}\right),\left(\alpha_{k, 2}, \alpha_{k, 3}\right), \ldots,\left(\alpha_{k, v_{k}-1}, \alpha_{k, v_{k}}\right)$. Hence, for each pair of consecutive intervals containing an angle $\theta=\theta_{k}^{*}+\frac{\mu}{N}, \mu \in \mathscr{Y}_{k}$, we get a contribution of at least 1 for the quantity $\mathcal{S}_{k}$.

Every element of $J_{k}$ can be written as $\theta_{k}^{*}+\frac{\mu}{N}$, for

$$
0 \leqslant \mu \leqslant(1-\sqrt{\delta}) N \Delta \leqslant N \Delta=2 \pi M
$$

The Lebesgue measure of the elements of $J_{k}$ for which $\mu \notin \mathscr{Y}_{k}$ is then bounded by

$$
\frac{1}{N} \lambda\left(\mathscr{Y}_{k}^{c}\right)=\frac{2 \pi M}{N} \lambda_{2 \pi M}\left(\mathscr{Y}_{k}^{c}\right)
$$

where $\lambda$ denotes the standard Lebesgue measure. Hence, if an interval $\left(\alpha_{k, v}, \alpha_{k, v+1}\right)$ has a length strictly greater than this bound, it necessarily contains some $\theta=\theta_{k}^{*}+\frac{\mu}{N}$ for which 
$\mu \in \mathscr{Y}_{k}$. For some $c^{\prime}>0$ depending only on $n$, this condition is implied by

$$
\alpha_{k, v+1}-\alpha_{k, v}>C^{\prime} \frac{M}{N} \delta^{-2}(\log N)^{-1 / 4}(\log M)^{1 / 2}
$$

We will say that $\left(\alpha_{k, v}, \alpha_{k, v+1}\right)$ is a roomy gap if this inequality is satisfied, and a narrow gap if

$$
\alpha_{k, v+1}-\alpha_{k, v} \leqslant C^{\prime} \frac{M}{N} \delta^{-2}(\log N)^{-1 / 4}(\log M)^{1 / 2}
$$

By the previous discussion, $\mathcal{S}_{k}$ is at least the number of pairs of consecutive roomy gaps among the intervals $\left(\alpha_{k, 1}, \alpha_{k, 2}\right),\left(\alpha_{k, 2}, \alpha_{k, 3}\right), \ldots,\left(\alpha_{k, v_{k}-1}, \alpha_{k, v_{k}}\right)$. If there is no narrow gap, the number of such pairs is $\left(v_{k}-2\right)_{+} \geqslant v_{k}-2$. Moreover, if among the intervals, we replace a roomy gap by a narrow gap, this removes at most two pairs of consecutive roomy gaps. Hence, we deduce, for all $k \in \mathscr{N}_{2}$, that

$$
\mathcal{S}_{k} \geqslant v_{k}-2-2 \psi_{k}
$$

where $v_{k}$ is the number of zeros of $Z_{X_{j_{k}}}$ in the interval $J_{k}$ and $\psi_{k}$ the number of narrow gaps among these zeros. Hence, we get the lower bound:

$$
\mathbb{E}_{\mathrm{SU}(N)}^{(n)}\left(\sum_{k \in \mathscr{N} / 2} \mathcal{S}_{k}\right) \geqslant \mathbb{E}_{\mathrm{SU}(N)}^{(n)}\left(\sum_{k \in \mathscr{N}_{2}} v_{k}-2 K-2 \psi\right)
$$

where $\psi$ is the total number of narrow gaps among the zeros in $[0,2 \pi)$ of all the functions $\left(Z_{j}\right)_{1 \leqslant j \leqslant n}$.

Now, $\mathbb{P}_{\mathrm{SU}(N)}^{(n)}$-a.s., for all $k \in \mathscr{N}_{2}$, we have

$$
\begin{aligned}
v_{k} & =\left|\left\{\theta \in\left[\theta_{k}^{*}, \theta_{k}^{*}+(1-\sqrt{\delta}) \Delta\right], Z_{j_{k}}(\theta)=0\right\}\right| \\
& \geqslant\left|\left\{\theta \in\left[\theta_{k}+\sqrt{\delta} \Delta, \theta_{k}+(1-\sqrt{\delta}) \Delta\right], Z_{j_{k}}(\theta)=0\right\}\right| \\
& =\frac{N(1-2 \sqrt{\delta}) \Delta}{2 \pi}+\frac{1}{\pi}\left(\operatorname{Im} \log Z_{X_{j_{k}}}\left(\theta_{k}+(1-\sqrt{\delta}) \Delta\right)-\operatorname{Im} \log Z_{X_{j_{k}}}\left(\theta_{k}+\sqrt{\delta} \Delta\right)\right) \\
& \geqslant \frac{N}{K}(1-2 \sqrt{\delta})-\frac{1}{\pi} \sum_{j=1}^{n}\left|\left(\operatorname{Im} \log Z_{X_{j}}\left(\theta_{k}+(1-\sqrt{\delta}) \Delta\right)-\operatorname{Im} \log Z_{X_{j}}\left(\theta_{k}+\sqrt{\delta} \Delta\right)\right)\right|,
\end{aligned}
$$

the second equality coming from Proposition 2.5. 
Adding this inequality for all $k \in \mathscr{N}_{2}$, and taking the expectation yields the estimate

$$
\begin{aligned}
& \mathbb{E}_{\mathrm{SU}(N)}^{(n)}\left(\sum_{k \in N_{2}} v_{k}\right) \geqslant \frac{N}{K}(1-2 \sqrt{\delta}) \mathbb{E}_{\mathrm{SU}(N)}^{(n)}\left(\left|\mathscr{N}_{2}\right|\right) \\
& \quad-\sum_{j=1}^{n} \sum_{k=0}^{K-1} \mathbb{E}_{\mathrm{SU}(N)}^{(n)}\left[\left|\left(\operatorname{Im} \log Z_{X_{j}}\left(\theta_{k}+(1-\sqrt{\delta}) \Delta\right)-\operatorname{Im} \log Z_{X_{j}}\left(\theta_{k}+\sqrt{\delta} \Delta\right)\right)\right|\right]
\end{aligned}
$$

and using (14) to estimate the terms on the right-hand side we therefore conclude that

$$
\begin{aligned}
\mathbb{E}_{\mathrm{SU}(N)}^{(n)}\left(\sum_{k \in \mathscr{N}_{2}} v_{k}\right) & \geqslant \frac{N}{K}(1-2 \sqrt{\delta}) K(1-O(\sqrt{\delta} \log (1 / \delta)))+O(K \sqrt{\log M}) \\
& \geqslant N(1-O(\sqrt{\delta} \log (1 / \delta)))+O\left(\frac{N \sqrt{\log M}}{M}\right) .
\end{aligned}
$$

It remains to estimate

$$
\mathbb{E}_{\mathrm{SU}(N)}^{(n)}[2 \psi]=2 n \mathbb{E}_{\mathrm{SU}(N)}[\chi]=2 n \mathbb{E}_{U(N)}[\chi]
$$

where $\chi$ denotes the number of narrow gaps between the eigenvalues of the canonical unitary matrix $X$. The replacement of $\mathrm{SU}(N)$ by $U(N)$ is possible since the notion of narrow gap is invariant by rotation of the eigenvalues. Note that this estimate of the number of narrow gaps is the analog of the assumption made by Bombieri and Hejhal on the proportion of small gaps between zeros of $L$-functions. Now, the last expectation can be estimated by the following result.

Lemma 3.15. For $N \geqslant 1$ and $\epsilon>0$, let $U$ be a Haar-distributed matrix on $U(N)$ and let $\chi_{\varepsilon}$ be the number of pairs of eigenvalues of $U$ whose argument differ by at most $\varepsilon / N$. Then, $\mathbb{E}\left[\chi_{\varepsilon}\right]=O\left(N \varepsilon^{3}\right)$.

Proof. For $\theta_{1}, \theta_{2} \in \mathbb{R}$, the two-point correlation density of the eigenvalues of $U$ at $\mathrm{e}^{\mathrm{i} \theta_{1}}$ and $\mathrm{e}^{\mathrm{i} \theta_{2}}$, with respect to the Haar probability measure on the unitary group, is given by

$$
\rho\left(\mathrm{e}^{\mathrm{i} \theta_{1}}, \mathrm{e}^{\mathrm{i} \theta_{2}}\right)=N^{2}\left[1-\left(\frac{\sin \left[N\left(\theta_{2}-\theta_{1}\right) / 2\right]}{N \sin \left[\left(\theta_{2}-\theta_{1}\right) / 2\right]}\right)^{2}\right] .
$$

Now,

$$
N\left|\sin \left[\left(\theta_{2}-\theta_{1}\right) / 2\right]\right| \leqslant N\left|\theta_{2}-\theta_{1}\right| / 2
$$


and then

$$
\left(\frac{\sin \left[N\left(\theta_{2}-\theta_{1}\right) / 2\right]}{N \sin \left[\left(\theta_{2}-\theta_{1}\right) / 2\right]}\right)^{2} \geqslant\left(\frac{\sin x}{x}\right)^{2}
$$

for $x=N\left(\theta_{2}-\theta_{1}\right) / 2$. Now, for all $x \in \mathbb{R},|\sin x| \geqslant \sin |x| \geqslant|x|-|x|^{3} / 6$, which implies

$$
\left(\frac{\sin x}{x}\right)^{2} \geqslant\left(1-\frac{x^{2}}{6}\right)^{2} \geqslant 1-\frac{x^{2}}{3}
$$

and

$$
\rho\left(\mathrm{e}^{\mathrm{i} \theta_{1}}, \mathrm{e}^{\mathrm{i} \theta_{2}}\right) \leqslant N^{2}\left[1-\left(\frac{\sin x}{x}\right)^{2}\right] \leqslant \frac{N^{2} x^{2}}{3}=\frac{N^{4}\left(\theta_{2}-\theta_{1}\right)^{2}}{6} .
$$

Integrating the correlation function for $\theta_{1} \in[0,2 \pi)$ and $\theta^{\prime}:=\theta_{2}-\theta_{1} \in[-\varepsilon / N, \varepsilon / N]$ gives

$$
\mathbb{E}\left[\chi_{\varepsilon}\right] \leqslant \int_{0}^{2 \pi} \frac{\mathrm{d} \theta}{2 \pi} \int_{-\varepsilon / N}^{\varepsilon / N} \frac{\mathrm{d} \theta^{\prime}}{2 \pi} \frac{N^{4}\left(\theta^{\prime}\right)^{2}}{6} \leqslant N^{4} \int_{-\varepsilon / N}^{\varepsilon / N}\left(\theta^{\prime}\right)^{2} \mathrm{~d} \theta^{\prime}=O\left(N^{4}(\varepsilon / N)^{3}\right) .
$$

From this result, applied for

$$
\varepsilon=C^{\prime} M \delta^{-2}(\log N)^{-1 / 4}(\log M)^{1 / 2}
$$

we get the estimate

$$
\mathbb{E}_{\mathrm{SU}(N)}^{(n)}[2 \psi]=O\left(N \varepsilon^{3}\right)=O\left(N M^{3} \delta^{-6}(\log N)^{-3 / 4}(\log M)^{3 / 2}\right)
$$

Substituting the estimates (25) and (26) into (24), and recalling that $M:=N / K$ so $2 K=O(N / M)$, we find that

$$
\mathbb{E}_{\mathrm{SU}(N)}^{(n)}\left(\sum_{k \in \mathscr{N}_{2}} \mathcal{S}_{k}\right) \geqslant N\left[1-O\left(\sqrt{\delta} \log (1 / \delta)+\frac{\sqrt{\log M}}{M}+M^{3} \delta^{-6}(\log N)^{-3 / 4}(\log M)^{3 / 2}\right)\right] .
$$

From the values taken for $\delta$ and $M$, we get

$$
\begin{aligned}
\sqrt{\delta} \log (1 / \delta) & =O\left((\log N)^{-3 / 64} \log \log N\right), \\
\frac{\sqrt{\log M}}{M} & =O\left(\sqrt{\log \log N}(\log N)^{-3 / 64}\right)
\end{aligned}
$$

and

$$
\begin{aligned}
M^{3} \delta^{-6}(\log N)^{-3 / 4}(\log M)^{3 / 2} & =O\left((\log N)^{9 / 64}(\log N)^{18 / 32}(\log N)^{-3 / 4}(\log \log N)^{3 / 2}\right) \\
& =O\left((\log N)^{-3 / 64}(\log \log N)^{3 / 2}\right)
\end{aligned}
$$


Finally, we get

$$
\mathbb{E}_{\mathrm{SU}(N)}^{(n)}\left(\sum_{k \in N_{2}} \mathcal{S}_{k}\right)=N\left(1-O\left((\log N)^{-1 / 22}\right)\right)
$$

which completes the proof of Theorem 1.1.

\section{Funding}

Y.B-A. was partially funded by the Schweizerischer Nationalfonds PDFMP2 134897/1.

\section{Acknowledgements}

We thank Brian Conrey and David Farmer for encouraging us to investigate this problem.

\section{References}

[1] Andrade, J. and J. Keating. “Conjectures for the integral moments and ratios of $L$-functions over function fields." Journal of Number Theory 142 (2014): 102-48.

[2] Bogomolny, E., O. Bohigas, and P. Leboeuf. "Quantum chaotic dynamics and random polynomials." Journal of Statistical Physics 85, no. 5-6 (1996): 639-79.

[3] Bombieri, E. and D. Hejhal. "On the distribution of zeros of linear combinations of Euler products." Duke Mathematical Journal 80, no. 3 (1995): 821-62.

[4] Bourgade, P., C. Hughes, A. Nikeghbali, and M. Yor. "The characteristic polynomial of a random unitary matrix: a probabilistic approach." Duke Mathematical Journal 145, no. 1 (2008): 45-69.

[5] Bourgade, P., J. Najnudel, and A. Nikeghbali. "A unitary extension of virtual permutations." International Mathematics Research Notices 2013, no. 18 (2013): 4101-34.

[6] Bump, D. and A. Gamburd. "On the averages of characteristic polynomials from classical groups." Communications in Mathematical Physics 265, no. 1 (2006): 227-74.

[7] Dehaye, P.-O. "Joint moments of derivatives of characteristic polynomials." Algebra and Number Theory 2, no. 1 (2008): 31-68.

[8] Diaconis, P. and M. Shahshahani. “On the Eigenvalues of Random Matrices." In Studies in Applied Probability, edited by J. Gan, 49-62. Journal of Applied Probability: Special Vol. 31A. Sheffield: Appl. Probab. Trust, 1994.

[9] Hughes, C. "On the characteristic polynomial of a random unitary matrix and the Riemann zeta function." University of Bristol, PhD thesis, 2001.

[10] Hughes, C., J. Keating, and N. O'Connell. “On the characteristic polynomial of a random unitary matrix." Communications in Mathematical Physics 220, no. 2 (2001): 429-51.

[11] Hughes, C. and A. Nikeghbali. "Zeros of random polynomials cluster uniformly near the unit circle." Compositio Mathematica 144, no. 3 (2008): 734-46. 
[12] Jacod, J., E. Kowalski, and A. Nikeghbali. "Mod-Gaussian convergence: new limit theorems in probability and number theory." Forum Mathematicum 23, no. 4 (2011): 835-73.

[13] Katz, N. and P. Sarnak. Random Matrices, Frobenius Eigenvalues and Monodromy, 1-26. American Mathematical Society Colloquium Publications 45. Providence, RI: American Mathematical Society, 1999.

[14] Katz, N. and P. Sarnak. "Zeros of zeta functions and symmetry." American Mathematical Society. Bulletin 36, no. 1 (1999): 1-26.

[15] Keating, J. and N. Snaith. "Random matrix theory and $\zeta(1 / 2+\mathrm{i} t)$. " Communications in Mathematical Physics 214, no. 1 (2000): 57-85.

[16] Keating, J. and N. Snaith. "Random matrix theory and $L$-functions at $s=1 / 2$." Communications in Mathematical Physics 214, no. 1 (2000): 91-110.

[17] Killip, R. and I. Nenciu. "Matrix models for circular ensemble." International Mathematics Research Notices 2004, no. 50 (2004): 2665-701.

[18] Kowalski, E. and A. Nikeghbali. "Mod-Gaussian convergence and the value distribution of $\zeta(1 / 2+i t)$ and related quantities." Journal of the London Mathematical Society. Second Series 86, no. 1 (2012): 291-319.

[19] Meckes, E. and M. Meckes. "Concentration and convergence rates for spectral measures of random matrices." Probability Theory and Related Fields 156, no. 1-2 (2013): 145-64.

[20] Mezzadri, F. and N. Snaith (editors). Recent Perspectives in Random Matrix Theory and Number Theory. London Mathematical Society Lecture Note Series 322. Cambridge: Cambridge University Press, 2005.

[21] Rudnick, Z. and P. Sarnak. "Zeros of principal $L$-functions and random matrix theory." Duke Mathematical Journal 81, no. 2 (1996): 269-322.

[22] Selberg, A. "Old and new conjectures and results about a class of Dirichlet series." Proceedings of the Amalfi Conference on Analytic Number Theory (Maiori, 1989), 367-85. Salerno: University of Salerno, 1992.

[23] Snaith, N. "Riemann zeros and random matrix theory." Milan Journal of Mathematics 78, no. 1 (2010): 135-52. 